\title{
EL YACIMIENTO ACHELENSE DE AS GÁNDARAS DE BUDIÑO: SÍN- TESIS Y PERSPECTIVAS DESPUÉS DE 50 AÑOS DE DESENCUENTROS
}

\author{
EDUARDO MÉNDEZ-QUINTAS ${ }^{(1)}$, MANUEL SANTONJA $^{(1)}$, ALFREDO PÉREZ-GONZÁLEZ $^{(1)}$, LEE J. \\ ARNOLD $^{(2)}$, JOÃO PEDRO CUNHA RIBEIRO ${ }^{(3)}$, MARTINA DEMURO ${ }^{(2)}$, MIKEL DÍAZ-RODRÍGUEZ $^{(4)}$, \\ MATHIEU DUVAL ${ }^{(5)}$, ALBERTO GOMES ${ }^{(6)}$, JOSÉ MEIRELES ${ }^{(7)}$, SÉRGIO MONTEIRO-RODRIGUES ${ }^{(8)} \&$ \\ ANDREA SERODIO DOMÍNGUEZ ${ }^{(9)}$
}

Resumen:

\begin{abstract}
El yacimiento paleolítico de As Gándaras de Budiño es un yacimiento clave en la Prehistoria antigua de la Península Ibérica. Aunque el yacimiento ha sido excavado en diferentes ocasiones, muchos de los aspectos geológicos y arqueológicos no son bien conocidos. Entre estos problemas está la cronología, que inicialmente fue atribuida al MIS 2 -originando una fuerte controversia-, aunque en la actualidad podemos afirmar que tiene una cronología del Pleistoceno Medio. Las industrias líticas son de tipo achelense de grandes lascas (LFA), con características similares a la de otros yacimientos de la Península Ibérica. Este artículo es un estado de la cuestión para el yacimiento, previo al reinicio de las investigaciones.
\end{abstract}

Palavras-chave: Paleolítico Inferior; Achelense; Pleistoceno Medio; Geoarqueología; Industria Lítica; LCT; Río Miño; Galicia

Abstract:

The Acheulean site of As Gándaras de Budiño: synthesis and perspectives after 50 years of disencouters

As Gándaras de Budiño (GB) is a key locality for the Lower Palaeolithic record of the Iberian Peninsula. Although the site has been excavated in different occasions over the last decades, several geological and archaeological aspects are still not well known. Among other issues, the age of the lithic assemblage has been debated for a long time: despite an initial correlation to the MIS 2, new evidence rather points towards a Middle Pleistocene age instead. The lithic assemblage characterized as large flake acheulean type (LFA), with many similarities with other acheulean sites in the Iberian Peninsula. This work presents an updated overview of the current state of knowledge for GB while a new multidisciplinary research project has been recently initiated

Keywords: Lower Paleolithic; Acheulean; Middle Pleistocene; Geo-archaeology; Lithic industry; LCT; Miño river; Galicia

\section{INTRODUCCIÓN}

El yacimiento paleolítico de As Gándaras de Budiño se encuentra situado en el interior de la cuenca del río Louro, uno de los últimos afluentes importantes del río Miño antes de su desembocadura en el mar Atlántico, y administrativamente en el ayuntamiento de O Porriño (Pontevedra, Galicia, España) (Fig. 1). Aunque protegido con la figura de Bien de Interés Cultural -BIC- (DOGA 137 del 21 de Julio de 1997), el yacimiento "clásico", que fue afectado inicialmente por explotaciones de arcillas, se encuentra actualmente muy mermado a causa del desarrollo de diferentes infraestructuras, entre ellas una autovía y un polígono industrial.

Esta localidad (GB en lo sucesivo) ha sido tradicionalmente la referencia más habitual a la vez que controvertida para el Paleolítico antiguo del NW peninsular. Su problemática interpretación derivaba de la atribución cronológica, basada en dataciones de ${ }^{14} \mathrm{C}$ en cronologías del MIS 2 (AGUiRRE \& BUTZER, 1967; BUTZER, 1967) que entraban en profunda contradicción con el neto carácter achelense de las industrias líticas recuperadas (ECHAIDE, 1971; VIDAL ENCINAS, 1983).

GB fue excavado en diferentes ocasiones a lo

\footnotetext{
${ }^{(1)}$ Unidad de Investigación Consolidada de la Junta de Castilla y León UIC 182. Centro Nacional de Investigación sobre la Evolución Humana (CENIEH). Paseo de Atapuerca, 3. 09002 Burgos, España.

${ }^{(2)}$ School of Physical Sciences, Environment Institute, and Institute for Photonics and Advanced Sensing (IPAS), University of Adelaide, North Terrace Campus, Adelaide SA 5005, Australia.

${ }^{(3)}$ Faculdade de Letras da Universidade de Lisboa e Centro de Arqueologia da Universidade de Lisboa (UNIARCH) e Lab2pt, 1600-214 Lisbon, Portugal.

${ }^{(4)}$ Grupo de Estudos para a Prehistoria do Noroeste Ibérico. Arqueoloxía, Antigüidade e Territorio (GEPN-AAT). Dpto de Historia, Universidade de Santiago de Compostela, Praza da Universidade, $n^{\circ} 1,15782$ Santiago de Compostela, España.

${ }^{(5)}$ Australian Research Centre for Human Evolution. Environmental Futures Research Institute, Griffith University, 170 Kessels Road Nathan, QLD 4111, Australia.

${ }^{(6)}$ Faculdade de Letras, Universidade do Porto; Centro de Estudos de Geografia e Ordenamento do Território (CEGOT). Via Panorâmica s/n, P 4150-564, Porto, Portugal

(7) Universidade do Minho; Lab2pt, 39 av. Central, 4700-Braga, Portugal

${ }^{(8)}$ Faculdade de Letras, Universidade do Porto; Centro de Investigação Transdisciplinar "Cultura, Espaço e Memória" (CITCEM). Via Panorâmica s/n, P 4150564, Porto, Portugal

${ }^{(9)}$ Data Gestión Cultural S.L. Avd. García Barbón 76 Galerías 36201 Vigo (Pontevedra) España
} 
Eduardo Méndez-Quintas, Manuel Santonja , Alfredo Pérez-González, Lee J. Arnold, João Pedro Cunha Ribeiro, Martina Demuro, Mikel Díaz-Rodríguez, Mathieu Duval, Alberto Gomes, José Meireles, Sérgio Monteiro-Rodrigues \& Andrea Serodio Domínguez

largo de los años, si bien con resultados solo parcialmente publicados (AGUIRRE, 1964; VIDAL ENCINAS, 1982A; CERQUEIRO LANDÍN, 1996). A pesar de tantas campañas de trabajo siguen sin resolverse los principales problemas, que tienen que ver con las condiciones estratigráficas y con la cronología. Afortunadamente en los últimos años se ha avanzado en el conocimiento tecnotipológico de la industria, lo que permite disponer de caracterizaciones sólidas (MÉNDEZ-QUINTAS, 2007, 2008; LOMBERA HERMIDA et al., 2011) que confirman el carácter plenamente achelense del yacimiento.

Con motivo del desarrollo del proyecto internacional "Minho/Miño, Ocupações pleistocénicas do Baixo Minho" (CUNHA RiBEIRo et al., 2017), que supone una nueva etapa de investigaciones multidisciplinares en el Paleolítico regional, en este artículo pretendemos aportar una síntesis actualizada de los conocimientos disponibles sobre GB, centrada especialmente en el ámbito geomorfológico. En el marco del proyecto Minho/Miño pretendemos en primer lugar avanzar en el conocimiento de las dinámicas sedimentarias existentes en el yacimiento, con objeto de profundizar en la definición de las condiciones tafonómicas que afectaron a la formación del registro arqueológico. Otro reto fundamental será conseguir óptimas cronologías numéricas. Para ello se tomarán muestras para datación por técnicas de Luminiscencia (pIR-IR y TT-OSL) y Resonancia Paramagnética Electrónica (ESR), que tan buenos resultados ha deparado en yacimientos regionales en contextos sedimentarios similares (MENDEZ-QUINTAS et al., 2018).

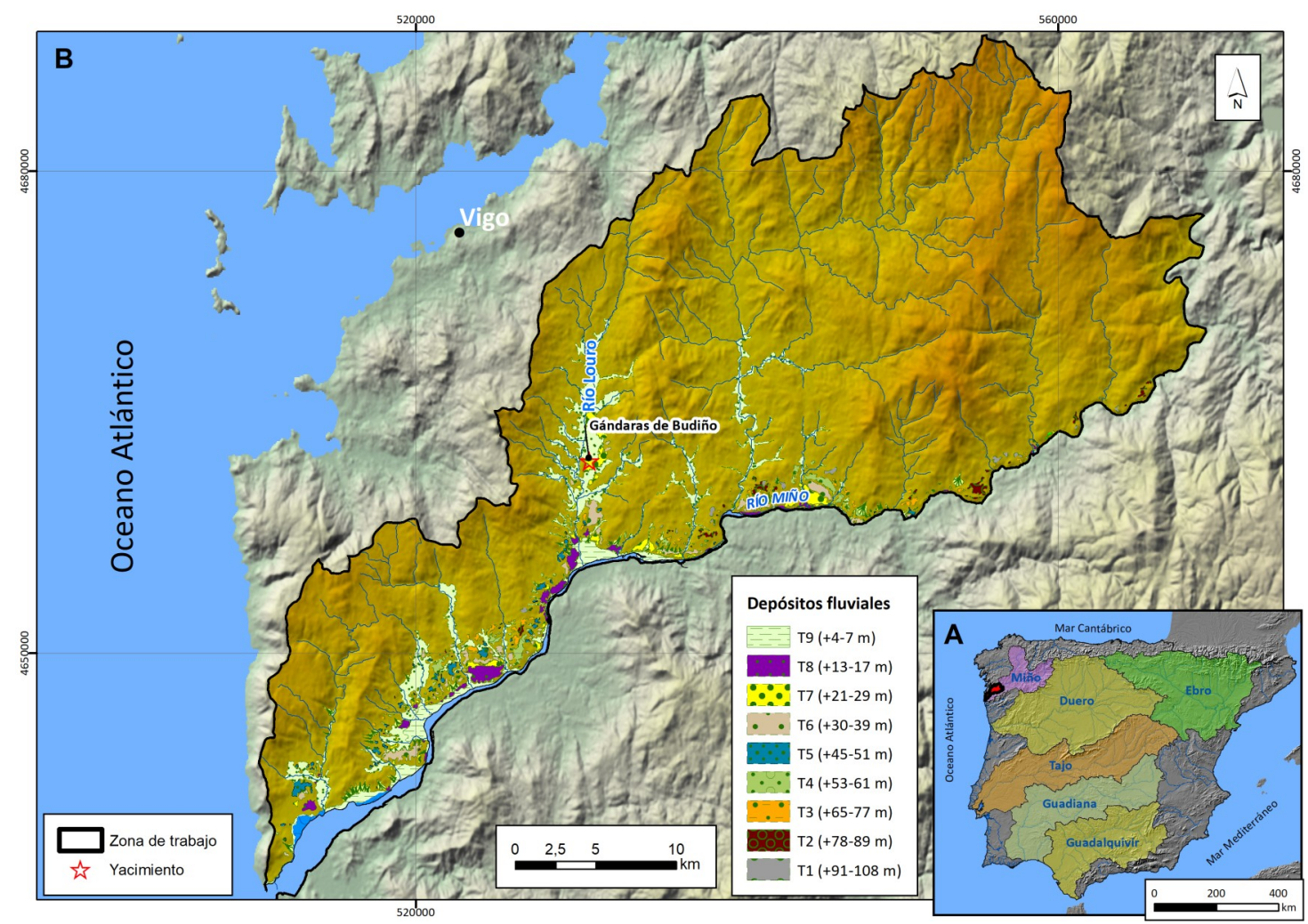

Fig. 1. A) Localización del sector español de la cuenca baja del río Miño en la Península Ibérica; B) Situación concreta del yacimiento.

Fig. 1. A) Location of the Spanish sector of the lower basin of the Miño River in the Iberian Peninsula; B) Location of the archaeological site.

\section{HISTORIA DE LAS INVESTIGACIONES}

GB fue descubierto por el geomorfólogo francés H. Nonn en 1961. En el curso de los trabajos de campo para su tesis doctoral identificó (NoNN, 1966, 1967) una serie de herramientas líticas de aspecto achelense en un nivel de arenas con gravas visible en los cortes de las explotaciones de arcilla existentes en la zona (Fig. 2). Nonn avisó a J. M. Álvarez Blázquez, por aquel entonces Comisario Local de Excavaciones de Vigo, quien desarrollaría intensas labores de prospección en la zona recuperando una importante colección de materiales. La importancia que presentaba el yacimiento llevó a Álvarez Blázquez a contactar con el paleontólogo E. Aguirre -a quien le unían vínculos familiares-, que se encargaría de acometer la primera excavación (NoNN, 1967).

En esas fechas Aguirre formaba parte del equipo de investigación que trabajaba en los yacimientos arqueo-paleontológicos mesopleistocenos de Ambrona y Torralba (Soria), dirigidos por el profesor F. 
C. Howell (Howell et al., 1995). Los primeros trabajos preliminares se llevaron a cabo en septiembre de 1962 y en el verano de 1963, con ayuda económica de la "Wenner-Gren Foundation for Anthropological Research", se emprendió la excavación del yacimiento (AGUIRRE, 1964) contando con la colaboración de investigadores de gran relevancia como P. Biberson, D. Collins, L. Freeman, F. Clark Howell y muy especialmente K. W. Butzer.

Las excavaciones de Aguirre supusieron la apertura de 16 zanjas de $1 \mathrm{~m}$ de ancho con una longitud total de $191 \mathrm{~m}$ (Fig.3). Era una estrategia inspirada en la que aplicaba Howell en Ambrona y Torralba, aunque en GB faltó excavar en extensión, como se hacía en los yacimientos sorianos a partir de las catas iniciales. La actuación de Aguirre permitió recuperar un importante conjunto de materiales líticos y tener una primera visión de los procesos geológicos que formaron el yacimiento. Las conclusiones alcanzadas proponían la existencia de "suelos de taller" y restos de "hogares" en el contexto de un nivel de naturaleza "coluvial" (AGUIRRE, 1964) (Fig.4).

Fig. 2. Fotografía con la posición estratigráfica y una muestra de las industrias líticas recuperadas por H. Nonn en el yacimiento de As Gándaras de Budiño (NonN 1966).

Fig. 2. Photograph with the stratigraphic position and a sample of the lithic assemblages recovered by H. Nonn in the As Gándaras de Budiño site (NONN 1966).
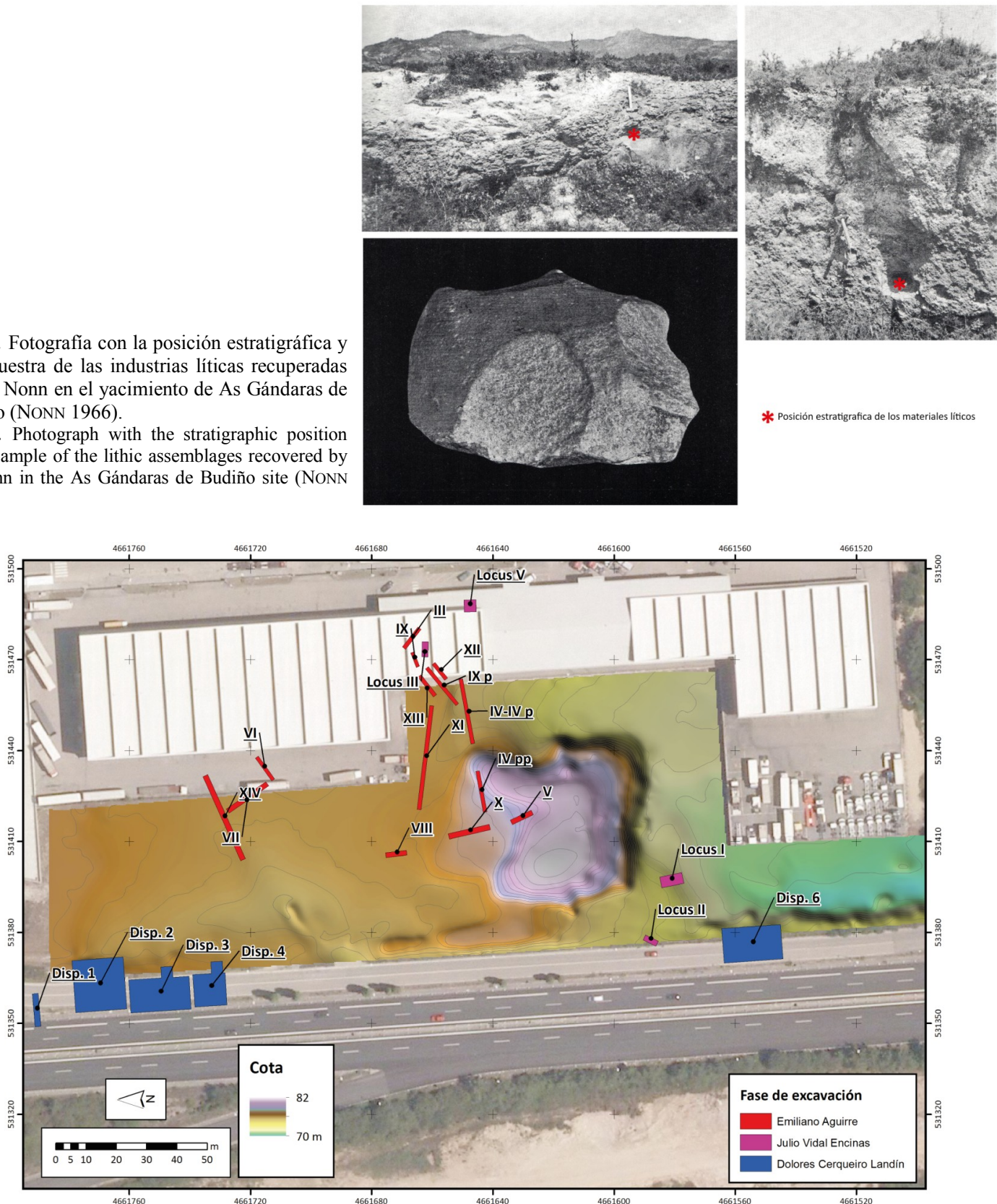

Fig. 3. Situación de las diferentes fases de excavación desarrolladas en el yacimiento.

Fig. 3. Location of the different phases of excavation carried out at the archaeological site. 

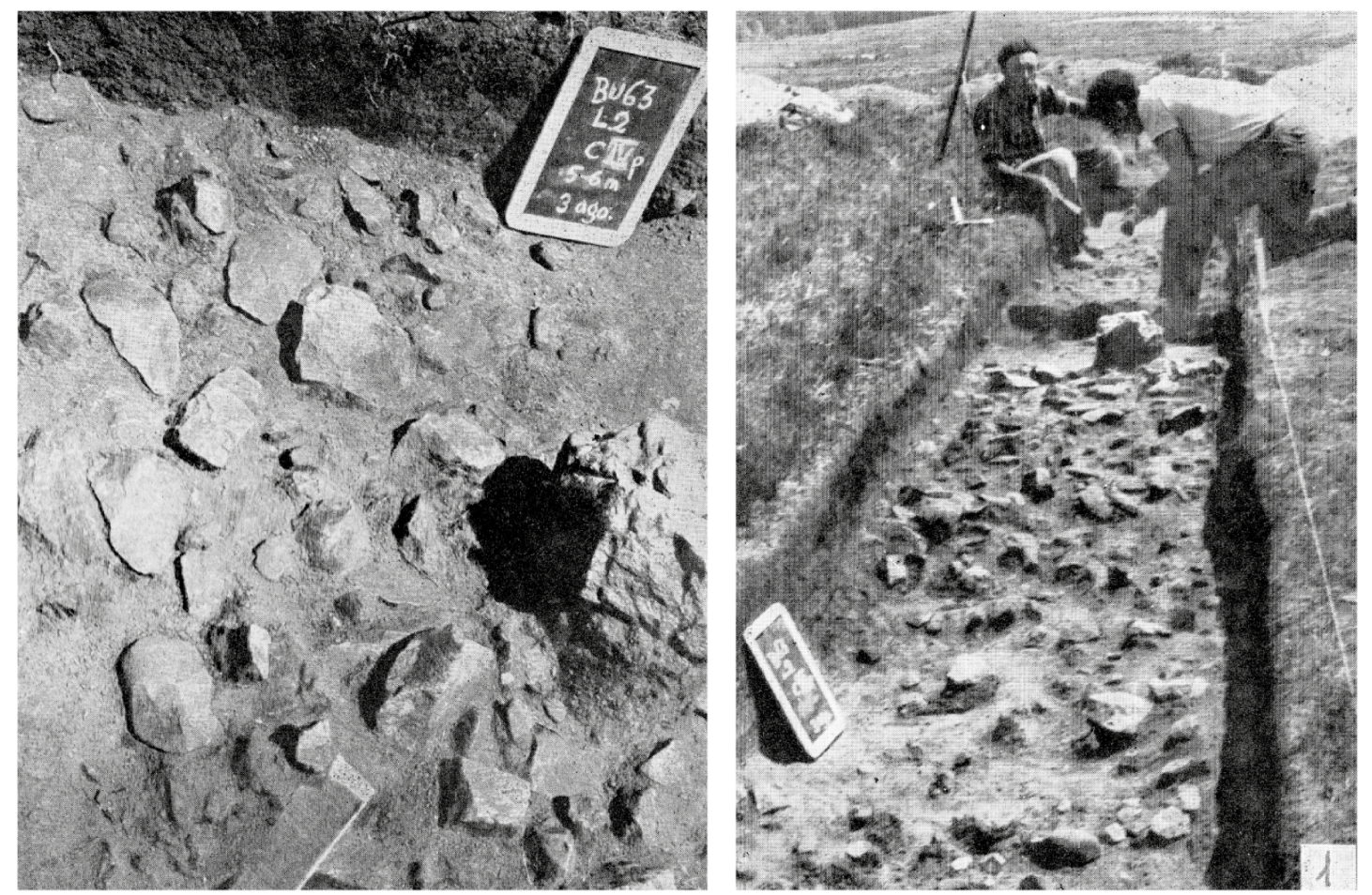

Fig. 4. Excavaciones de Aguirre, donde se observan las importantes concentraciones de materiales líticos, interpretados como "suelos de taller" (AGUIRRE 1964).

Fig. 4. Excavation carried out by Aguirre, where the important concentration of lithics were observed, and interpreted as "knapping floors" (AGUIRRE 1964).

Las industrias recuperadas, unas 773 piezas, eran claramente achelenses, con típicos bifaces y hendedores, y alcanzaban densidades de hasta 31,7 piezas por $\mathrm{m}^{2}$ en la trinchera IVp. Estaban realizadas en cuarcita alóctona y cuarzo local, roca en la que se consideraba complicado identificar los sistemas de talla "por su fractura irregular y la consiguiente falta de tipismo en el lascado" (AGUIRRE, 1964). Poco después de aparecer publicada la monografía sobre el yacimiento, en 1967, saldrán sendos artículos firmados por Butzer y Aguirre en las revistas Science y Eiszeitalter und Gegenwart que discutían la problemática que la anómala cronología obtenida planteaba en relación con el arcaísmo de la industria lítica (AGUIRRE \& BUTZER, 1967; BUTZER, 1967).

Aunque en las primeras publicaciones de Aguirre ya teníamos una aproximación a las características de los materiales líticos, habría que esperar hasta 1971 para que viese la luz el primer estudio tecnológico específico realizado por D. Echaide, investigadora que también participaba de manera destacada en las excavaciones de Ambrona y Torralba y había colaborado con Aguirre en las campañas de GB (ECHAIDE, 1971). Este estudio confirmaba la presencia de elementos típicamente achelenses y atribuía la presencia de artefactos con cierto carácter arcaico a la materia prima empleada. En todo caso el aparente arcaísmo contrastaba con el acabado cuidado de los LCTs, lo cual per- mitía a Echaide situar la serie de GB dentro del "Achelense superior" (ECHAIDE, 1971).

Las investigaciones de campo en el yacimiento se detendrían hasta el año 1980, fecha en que J. M. Vidal Encinas retomaría las investigaciones desde el Institut du Quaternaire de Bordeaux bajo la supervisión científica de F. Bordes, desarrollando 3 campañas de excavación entre 1980 y 1982. Estos trabajos contaron también con la colaboración de J. P. Texier, que amplió a la región el estudio geológico. Las excavaciones abarcaron una superficie total de $70 \mathrm{~m}^{2}$, distribuidas en cuatro zonas diferentes: Locus I, II, III y V, mientras que en el Locus IV se recogieron varias piezas en posición estratigráfica, pero no se llegó a excavar en extensión; los Locus III y V (Fig. 3 y 5) se encontraban en las proximidades de las trincheras de Aguirre (TEXIER \& VIDAL ENCINAS, 1981; VIDAL ENCINAS, 1982a). En todas las zonas excavadas se recuperaron materiales procedentes de niveles estratigráficos diferentes asociados a facies sedimentarias fluviales y “coluviales". Los Locus I, II y III fueron excavados en 1980 y 1981 y depararon materiales asociados tanto a niveles "coluviales" como fluviales, sobre una superficie de $49 \mathrm{~m}^{2}$ (Fig. 5 . 2-3). La excavación del Locus V se realizó en 1982 en una superficie de $21 \mathrm{~m}^{2}$ (Fig. 5. 1), pero únicamente se obtuvieron industrias asociadas a los niveles de génesis "coluvial" (VIDAL ENCINAS, 1982A; MÉNDEZ-QUINTAS, 2008). 

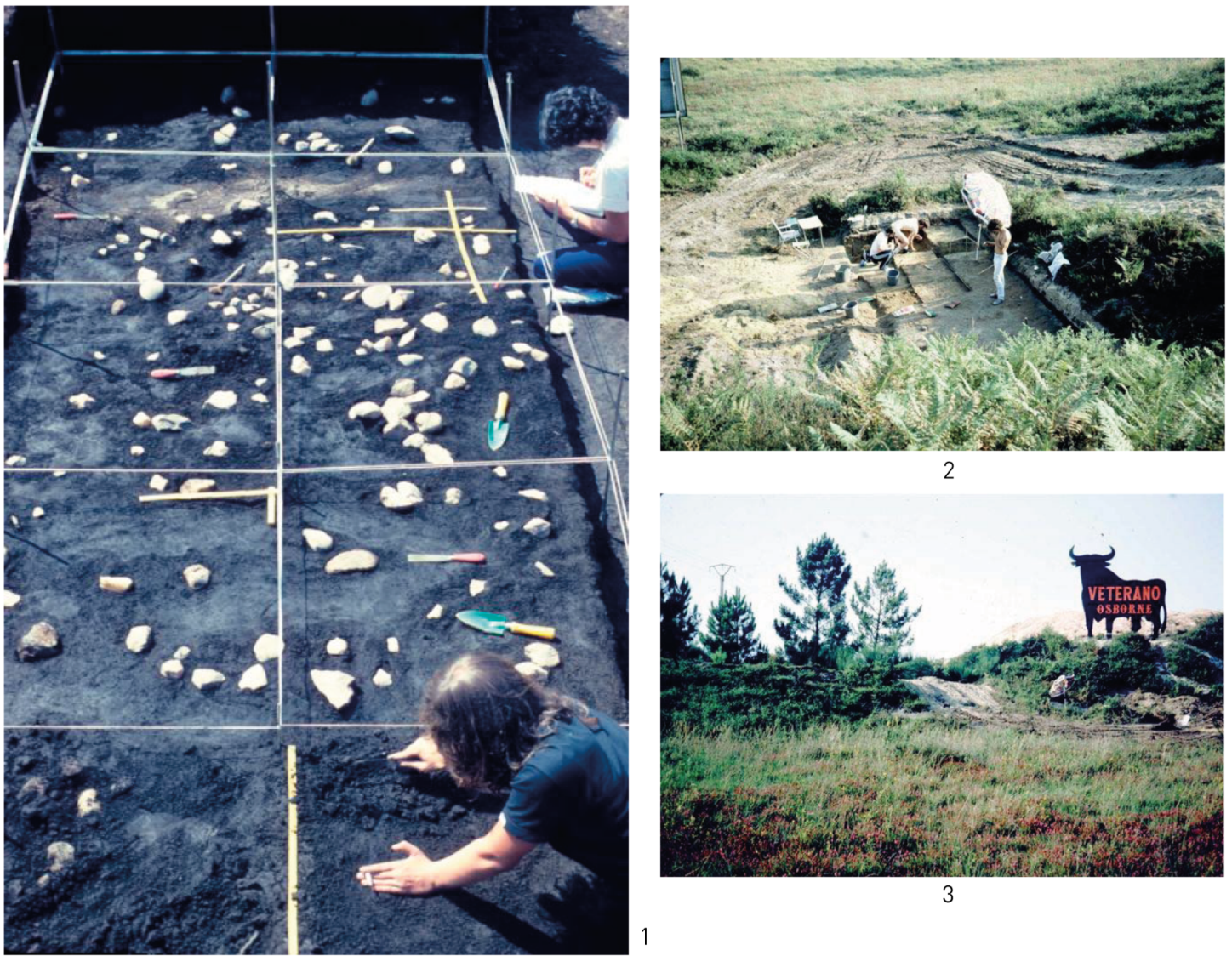

Fig. 5. Trabajos de excavación de Vidal Encinas. 1: excavación en el Locus V y 2-3: trabajos en el Locus I. Cortesía de J. M. Vidal Encinas.

Fig. 5. Excavation carried out by Vidal Encinas. 1: excavation of the locus V and 2-3: working at Locus I. Courtesy of J. M. Vidal Encinas.

Los resultados de esta intervención quedaron en su mayor parte inéditos, pero son a nuestro modo de ver los más completos y con mejor enfoque metodológico entre todos los desarrollados en Budiño. En las únicas publicaciones de índole preliminar realizadas por Vidal Encinas se hace una primera aproximación a la tecnología de los materiales recuperados, resaltando sus netas características achelenses. Asimismo, descarta la atribución cronológica defendida por Aguirre y Butzer y plantea una cronología más adecuada y acorde con los planteamientos académicos del momento (TEXIER \& VIDAL ENCINAS, 1981; VIDAL ENCINAS, 1982a, b).

Los últimos trabajos amplios de los que tenemos constancia se efectuaron a comienzos de la década de los 90. A diferencia de los precedentes, esta intervención no estaba fundamentada en necesidades de investigación, sino que se enmarcaba dentro de una actuación preventiva, ya que el yacimiento se iba a ver afectado por la construcción de una autovía y el desarrollo de un polígono industrial. Esta actuación fue dirigida por D. Cerqueiro Landín en colaboración con $\mathrm{M}^{\mathrm{a}} \mathrm{L}$. Pérez Rodríguez y A. López-Felpeto Gómez, desarrollándose entre los años 1991 y 1992 (CERQUEIRO LANDÍN, 1996). A pesar de ser la excavación que abarcó mayor extensión y contó con mayores recursos económicos, es la que menos volumen de información ha aportado, dándose incluso la paradoja de que los materiales recuperados no se encuentran depositados en ningún museo de titularidad pública.

Ante el riesgo que suponía la construcción de la autovía realizaron una amplia prospección en la zona, sondeos mecánicos con la finalidad de tener un mejor conocimiento de la secuencia estratigráfica y se excavaron unos $831 \mathrm{~m}^{2}$ en solo 2 campañas, repartidos en 5 sectores diferentes (Fig. 3). Según los parcos datos publicados (CERQUEIRO LANDíN, 1996) parece que se constataron diferencias estratigráficas entre estos sectores, si bien la impresión que prevalece es que las secuencias halladas eran semejantes a las descritas anteriormente.

Los materiales obtenidos no fueron demasiado numerosos si tenemos en cuenta la gran superficie excavada, entre las mayores para un yacimiento paleolítico en toda España, contabilizándose unos 4000 efectivos (4,8 piezas por $\mathrm{m}^{2}$ aproximadamente). En esta colección parecen predominar los elementos de producción (lascas y núcleos) junto a porcentajes representativos de LCTs.

En fecha posterior, a comienzos de los 2000, se ha desarrollado algún sondeo, seguimientos y 
Eduardo Méndez-Quintas, Manuel Santonja, Alfredo Pérez-González, Lee J. Arnold, João Pedro Cunha Ribeiro, Martina Demuro, Mikel Díaz-Rodríguez, Mathieu Duval, Alberto Gomes, José Meireles, Sérgio Monteiro-Rodrigues \& Andrea Serodio Domínguez

controles arqueológicos también en el contexto de acciones de arqueología preventiva, motivados por el desarrollo urbanístico de un polígono industrial, pero no se dispone de información precisa acerca de posibles resultados.

\section{ASPECTOS GEOMORFOLÓGICOS, SEDIMENTARIOS Y CRONOLOGÍA}

\subsection{Características geomorfológicas}

La interpretación de la estratigrafía ha sido uno de los puntos más controvertidos en $\mathrm{GB}$, ya que en torno a ella se ha movido la discusión sobre su atribución cronológica (AGUIRRE \& BUTZER, 1967; BUTZER, 1967; TEXIER \& VIDAL ENCINAS, 1981; VIDAL ENCINAS, 1982A; TEXIER, 1983-1984; GRACIA PRIETO et al., 2004).

El Louro, valle en el que se sitúa el yacimiento, drena unos $148,7 \mathrm{~km}^{2}$ y desarrolla un curso con una longitud próxima a $27 \mathrm{~km}$ que salva un desnivel de $170 \mathrm{~m}$. El río, con canal único y carga mixta de arenas y gravas, presenta un trazado poco sinuoso $(\mathrm{S}=0,84)$, de marcada dirección N-S (Fig. 6), adaptándose perfectamente a la llamada "Depresión Meridiana" o "Accidente Meridiano Gallego", una fosa de origen tectónico que se extiende desde Braga hasta Finisterre y marca el límite entre el bloque costero y los relieves de la "Dorsal Gallega" (SoS BAYNAT, 1965; NONN, 1966; DE ViCENTE \& Ve-
GAS, 2009; DE VICENTE et al., 2011).

Esta gran falla tardivarisca ha provocado el hundimiento del sustrato cristalino paleozoico (granitos, gneises y paragneises), y la creación de una amplia cuenca endorreica rellenada a lo largo del Neógeno (Sos Baynat, 1965; Nonn, 1966). En relación con esta dinámica tectónica se identifican otras subcuencas en la región: la del Louro al $\mathrm{N}$ del Miño, hacia el S la de Valença do Miño-Vila Nova de Cerveira y cara al E, la de Salvaterra de MiñoMonção. Las dos últimas con retazos de rellenos desconectados muy afectados por el proceso de encajamiento pleistoceno del Miño.

La subcuenca del Louro, menos erosionada, se conserva mejor. En su interior se identifica una potente acumulación de arcillas y arenas con intercalaciones de lignitos con más de $50 \mathrm{~m}$ de espesor en algunos puntos. Las asociaciones paleoflorísiticas y la magnetoestratigrafía permiten situar este relleno entre el Oligoceno Superior y el Mioceno (NONN \& Medus, 1963; MARTín-SERRANO \& Molina, 2005; VIEIRA et al., 2011). La cuenca se vio afectada por episodios de subsidencia derivados de actividad neotectónica o del propio asentamiento del fondo de la depresión; se constatan fallas en el tramo superior de los rellenos miocenos que no parecen afectar a los depósitos pleistocenos (SOS BAYNAT, 1965; NONN, 1966; BUTZER, 1967; GRACIA PRIETO et al., 2004). Aunque con los datos actuales la reconstrucción de la evolución final de la cuenca es un tanto especulativa, parece posible que durante buena parte

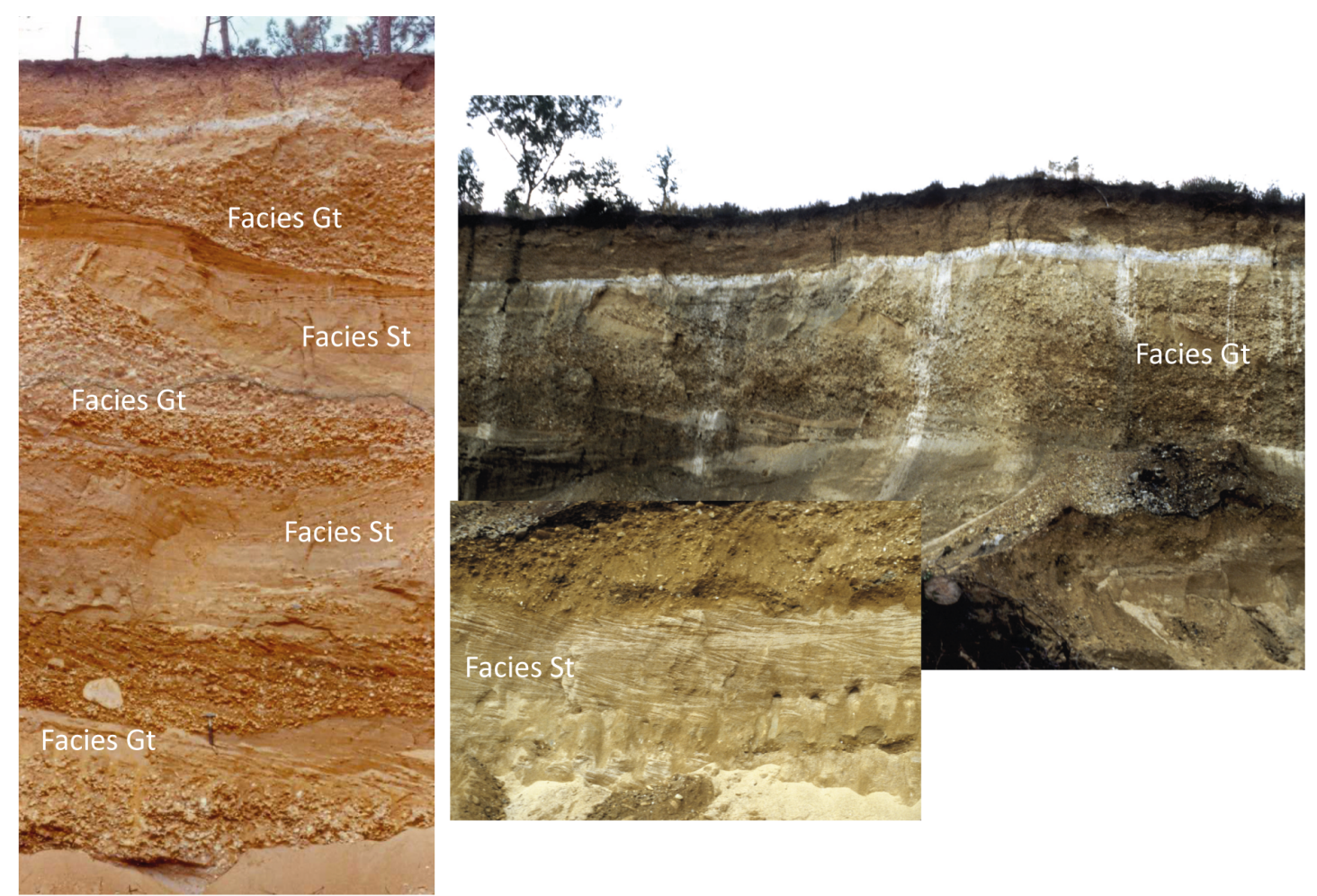

Fig. 6. Terraza T6 (+30-39 m) en Albeos, donde se puede apreciar la disposición de las facies de tipo Gt y St.

Fig. 6. Terrace T6 $(+30-39 \mathrm{~m})$ in Albeos, where the position of the Gt and $S t$ facies can be observed. 
del Pleistoceno habría seguido manteniendo carácter endorreico (GRACIA PRIETO et al., 2004).

Este aislamiento de la depresión del Louro cambió a lo largo del Pleistoceno al ser capturada por el río Miño. Se puede asegurar que el proceso de captura se desarrolló con anterioridad al emplazamiento de la terraza T6 (+30-39 m), ya que es esta la primera que se reconoce claramente en el interior de la cuenca. Asimismo, también es concordante con el proceso de captura la morfología de los niveles estratigráficos presentes en la T6 en la zona $\mathrm{S}$ de la cuenca con amplias secuencias de más de 10 metros en las que alternan facies tipo $G p, G t$, St y $S p$ (Miall, 1996) (Fig. 6).

En relación con los sucesivos cambios del nivel de base regional comenzaron a producirse continuos procesos de encajamiento de la red fluvial, paralelos a los experimentados por el colector principal. Como testimonio de estos procesos, en la zona más meridional de la cuenca se conservan secuencias de terrazas en ambas orillas del río. En unos casos estas terrazas corresponden a depósitos aportados por el Louro y en otros a los aportes del Miño. Las respectivas cargas litológicas permiten reconocer su origen. El Louro transportó arenas y gravas de cuarzo, mientras que en los depósitos generados por el Miño la cuarcita es la litología dominante. Entre los diferentes autores no existe consenso con respecto al número y cotas de las terrazas reconocidas en la zona, barajándose la presencia de 4 niveles escalonados, sobre la llanura de inundación, con cotas relativas de $+63 \mathrm{~m},+55$ $\mathrm{m},+33 \mathrm{~m} \mathrm{y}+24 \mathrm{~m}$ (SOS BAYNAT, 1965; NONN, 1966; BUTZER, 1967; TEXIER \& VIDAL ENCINAS, 1981; TEXIER, 1983-1984; GRACIA PRIETO et al., 2004). Las últimas revisiones identifican en el entorno de GB dos niveles de terraza sobre la llanura de inundación, con cota y formación que corresponden con las T6 y T7 del Miño. El yacimiento se relacionaría con la más baja de las dos, la $\mathrm{T} 7$, con una cota relativa de $+23 \mathrm{~m}$ sobre el bankfull del Miño y de $+13 \mathrm{~m}$ sobre el del Louro (Fig. 7). Los depósitos fluviales asociados a las terrazas del Louro se caracterizan estratigráficamente por niveles de arenas con facies tipo $S p$ y $S t$ (estratificación planar o cruzada curva), alternando con niveles más detríticos, con elementos de tamaño grava (2$64 \mathrm{~mm})$ y canto $(64-256 \mathrm{~mm}$ ) en facies $G m, G p$ y $G t$. Este tipo de facies son características de ríos con modelos sedimentarios braided de arenas y baja sinuosidad (MIALL, 1996). Sobre estos materiales fluviales o directamente sobre los niveles miocenos se desarrollaron otras series de continuidad lateral variable y constantes cambios de facies. Estas unidades, definidas a veces en la bibliografía como "coluviales" (BUTZER, 1967; VIDAL ENCINAS, 1982a), responden más propiamente a facies distales de pequeños abanicos aluviales, difíciles de identificar dada la profunda alteración antrópica de la zona. La formación de estos depósitos res- ponde a fenómenos de removilización y redeposición de los sedimentos precedentes y/o a los aportes de las vertientes inmediatas, afectados por procesos edáficos de diferente entidad (MÉNDEZQUINTAS, 2007, 2008).

\subsection{Estratigrafía}

Los investigadores que han trabajado en GB aunque empleando terminologías diferentes han descrito una secuencia esencialmente idéntica. La estratigrafía arrancaría en los depósitos arcósicos miocenos, sobre los que se emplazan erosivamente paquetes de arenas medias y gruesas con gravas de cuarzo (facies $S t$ ) de la terraza fluvial T7 de $+23 \mathrm{~m}$. Salvo en los trabajos de Aguirre, estos niveles han sido excavados en extensión y han aportado importantes series de industria lítica. Se trata en esencia del único conjunto de sedimentos con evidencias directas de ocupación humana pleistocena en la zona clásica del yacimiento (MÉNDEZ-QUINTAS, 2007, 2008).

Sobre ellos y erosionándolos hasta hacerlos desaparecer localmente, se documentan dos grandes series de aportes laterales con facies tipo Gm o $\mathrm{Sm}$ ("coluviales" en la bibliografía), uno inferior con gravas y cantos matriz soportados por arenas gruesas y otro superior de arenas-limosas y menor contenido detrítico (BUTZER, 1967; TEXIER \& VIDAL ENCINAS, 1981; VIDAL ENCINAS, 1982A; TEXIER, 1983-1984) (Fig. 8-9). Ambos ciclos deposicionales aportan cantidades dispares de materiales arqueológicos, distribuidos y organizados según la topografía del terreno. Nos encontraríamos ante industrias recicladas de los eventos fluviales inferiores, granoseleccionadas y redepositadas desde su posición original. Estamos pues ante materiales claramente alóctonos que en ningún caso es posible interpretar cómo ocupaciones humanas comparables a las documentadas en facies fluviales, en especial con las que se han podido registrar en medios de baja energía. Como veremos, además de la historia geológica y los procesos sedimentarios de estos niveles las propias características de la industria aseguran esta interpretación (MÉNDEZ-QUINTAS, 2007, 2008).

\subsection{Cronología}

Como ya se ha apuntado la datación ha sido uno de los aspectos más controvertidos del yacimiento. Nonn fue el primero en plantear una cronología normal, dentro de los parámetros empleados en aquella época. Situaba los materiales del nivel de arenas y gravas dentro de los estadios templados asociados a las glaciaciones alpinas del Mindel o Riss (Nonn \& Medus, 1963; Nonn, 1966). Para hacerlo se apoyaba en la posición morfológica de 
Eduardo Méndez-Quintas, Manuel Santonja, Alfredo Pérez-González, Lee J. Arnold, João Pedro Cunha Ribeiro, Martina Demuro, Mikel Díaz-Rodríguez, Mathieu Duval, Alberto Gomes, José Meireles, Sérgio Monteiro-Rodrigues \& Andrea Serodio Domínguez
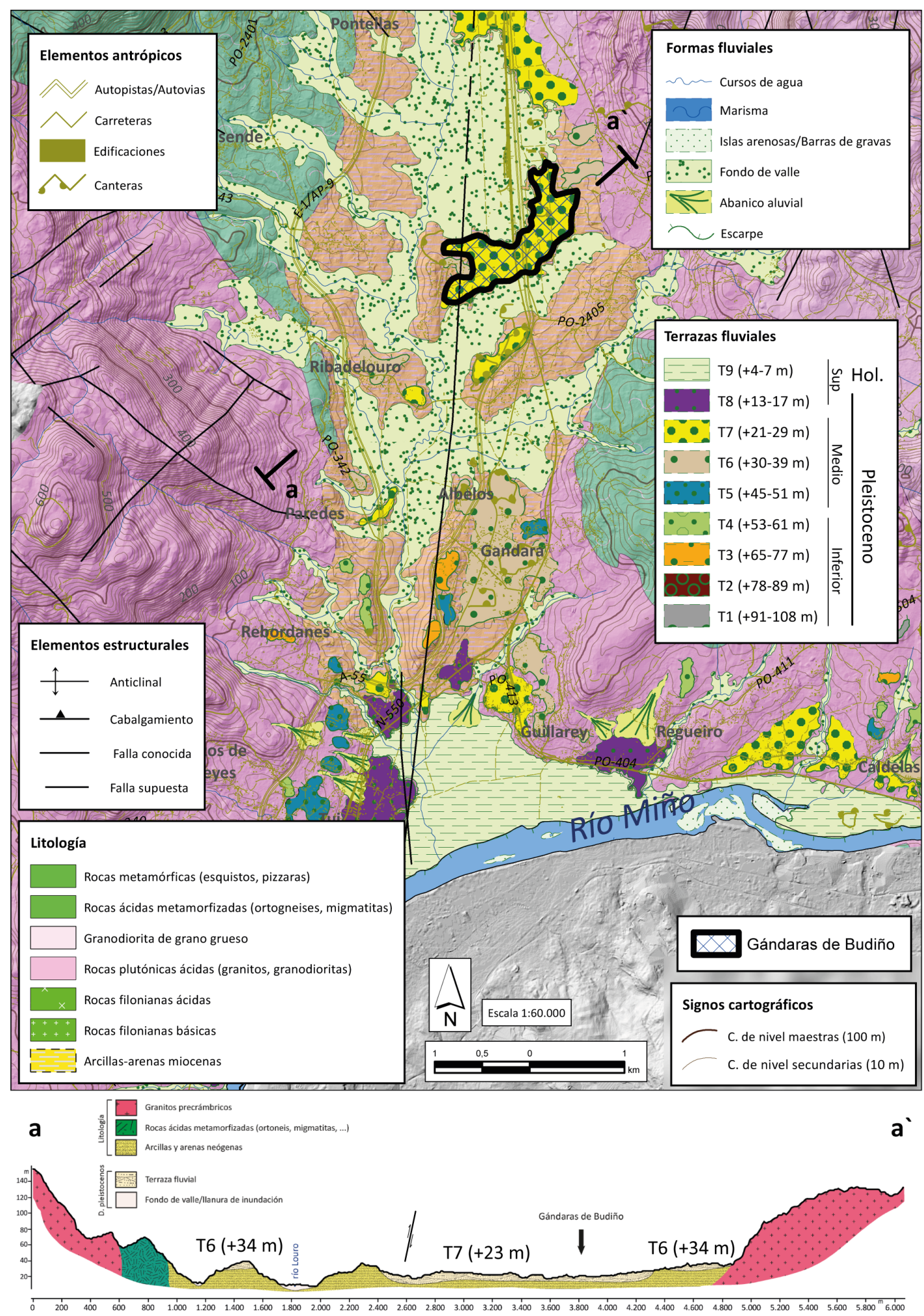

Fig. 7. Esquema y perfil geomorfológico del tramo final del valle del Louro hasta la confluencia con el Miño. Fig. 7. Geomorphological scheme and profile of the final sector of the Louro valley to the confluence with the Miño. 


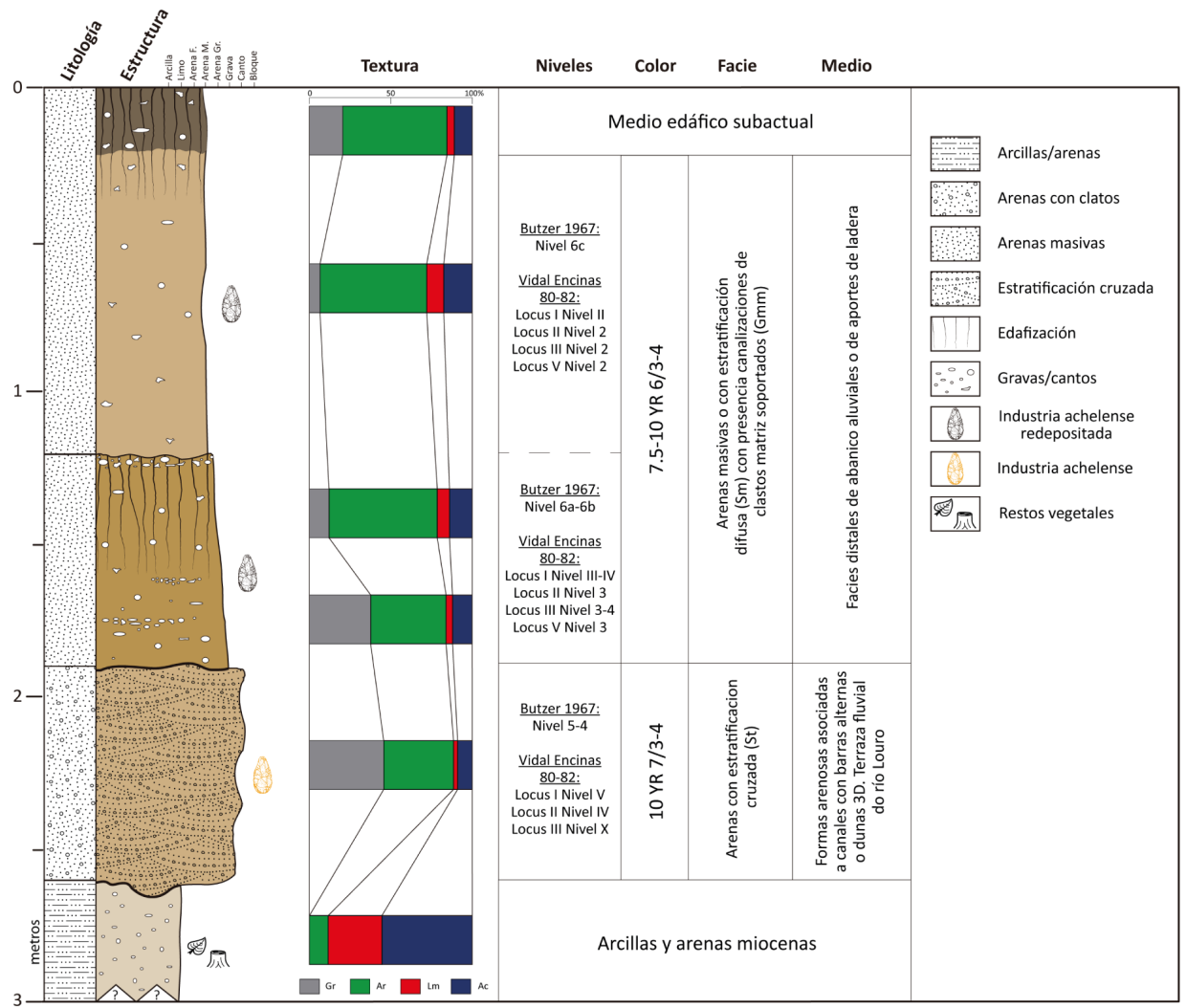

Fig. 8. Columna estratigráfica de la sección clásica del yacimiento (datos texturales extraídos de BUTZER 1967). Fig. 8. Stratigraphic column of the classical section of the archaeological site (textural data extracted from Butzer 1967).

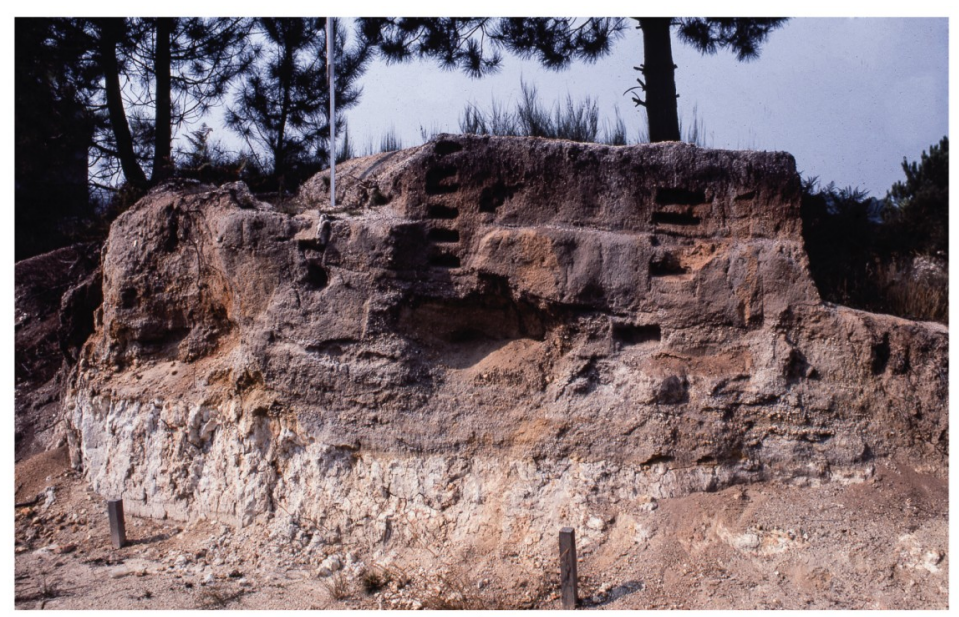

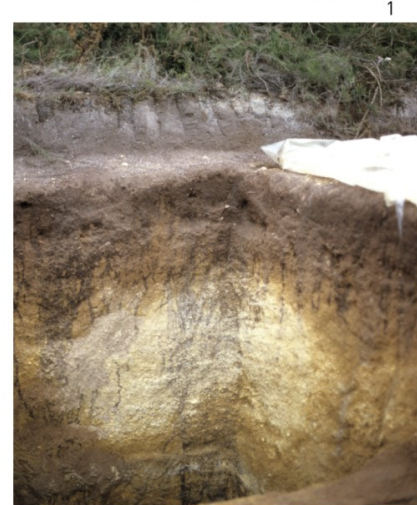

2

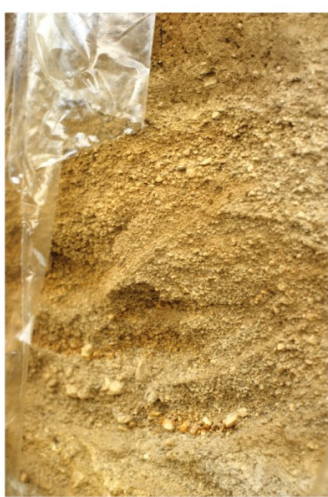

3
Fig. 9. Vistas de la secuencia estratigráfica del yacimiento durante las intervenciones de Vidal Encinas: 1. Vista del Locus III (cortesía de R. Patiño), 2-3. Locus I y detalle de los niveles fluviales del Locus II.

Fig. 9. View of the stratigraphic sequence of the archaeological site during the excavation carried out by Vidal Encinas: 1. View of the Locus III (courtesy of R. Patiño), 2-3. Locus I and detail of the fluvial levels of Locus II. 
Eduardo Méndez-Quintas, Manuel Santonja, Alfredo Pérez-González, Lee J. Arnold, João Pedro Cunha Ribeiro, Martina Demuro, Mikel Díaz-Rodríguez, Mathieu Duval, Alberto Gomes, José Meireles, Sérgio Monteiro-Rodrigues \& Andrea Serodio Domínguez

los depósitos, las características de la industria y en un primer estudio polínico que reconocía condiciones templadas y más secas que las actuales (NONN \& MEDUS, 1963; NonN, 1967).

Tras los trabajos de Aguirre y la posterior publicación de las interpretaciones geológicas de Butzer se presentarían las primeras dataciones numéricas por ${ }^{14} \mathrm{C}$ convencional para el yacimiento (AGUiRRe \& BUTZER, 1967; BUTZER, 1967). La aplicación de este método de datación para fechar industrias achelenses se puede considerar desconcertante, al margen de que se estaban fechando estratos con materiales en posiciones derivadas. Las muestras de carbón sometidas a datación procedían del llamado "Coluvión Inferior" (nivel 6a de Butzer) y se consideraban originadas en pretendidos hogares. Los resultados depararon, como era esperable, valores aberrantes, entre 28-19 ka en fechas calibradas, que unidos a una deficiente interpretación geoestratigráfica daría lugar a propuestas profundamente anómalas para el Paleolítico inferior de Galicia (VÁZQUEZ VARELA, 1973; FREEMAN, 1976; VV.AA, 1979; VÁZQUeZ VARELA, 1984; JORDÁ CERDÁ, 1986; CANO PAN, 1993), la lógica se volvería a imponer. Vidal Encinas propondría una cronología en base a criterios geológicos dentro de los límites del Pleistoceno medio, en relación con la glaciación del Riss (VIDAL ENCINAS, 1982a, b) y a partir de ahí esa sería la conclusión más aceptada por otros autores (SANTONJA \& QUEROL, 1984: 65; SANTONJA \& VILLA, 1990: 55; RAPOSO \& SANTONJA, 1995).

Nuevos elementos permiten en la actualidad realizar una aproximación más concreta a la cronología de las ocupaciones humanas de GB. Las fechas de ${ }^{14} \mathrm{C}$ obtenidas por Butzer y Aguirre podrían relacionarse con el momento de la deposición del llamado "Coluvión Inferior", ahora bien, el momento de la obtención de las fechas (años 60) también desaconseja emplearlas como indicador cronológico de este proceso. En cualquier caso, para los niveles fluviales que contienen la industria, podemos atenernos a las propuestas recientemente esbozadas para la secuencia de terrazas fluviales de la cuenca del Miño (VIVEEN et al., 2012; MÉNDEZ QUINTAS et al., 2017; MENDEZ-QUINTAS et al., 2018), de acuerdo con ellas las ocupaciones asociadas a la T3 se situarían en torno al MIS 7-6. Esta hipótesis deberá ser verificada mediante los resultados de futuras dataciones para dichos niveles fluviales, apoyadas en la revisión geoestratigráfica del yacimiento.

\section{CARACTERÍSTICAS TECNOECONÓ- MICAS Y TECNOTIPOLÓGICAS DE LOS MATERIALES LÍTICOS}

Las investigaciones realizadas en GB que hemos expuesto han permitido bien por los trabajos de excavación, bien por recogidas superficiales, acumular en diferentes instituciones museísticas y colecciones particulares ${ }^{1}$ un ingente conjunto de materiales líticos, que puede ascender a unas 20.000 piezas. A pesar de que las diferentes problemáticas de estas colecciones, derivadas fundamentalmente de sus procedencias estratigráficas y de cómo fueron formadas, se puede considerar que integran un conjunto esencialmente homogéneo en lo que se refiere a sus características tecnoeconómicas y tecnotipológicas. En general prevalecen los elementos asociados a la fase de producción (lascas, núcleos y chunks), con una representación menos significativa de configurados, que sin embargo dominan en las series de superficie, tales como las colecciones Álvarez Blázquez o Pelayo Fernández, integradas por piezas más seleccionadas (Tabla 1).

Tabla 1. Efectivos líticos desglosados por categorías tecnotipológicas para las principales colecciones de materiales. Tabla 1. Total amount of lithics of the main collections distributed by technotipological categories.

\begin{tabular}{|c|c|c|c|c|c|c|}
\hline \multirow{2}{*}{$\begin{array}{c}\text { Colección } \\
\text { Procedencia }\end{array}$} & \multicolumn{2}{|c|}{ E. Aguirre/D. Echaide } & \multicolumn{2}{|c|}{ J. Vidal Encinas } & \multirow{2}{*}{$\begin{array}{c}\text { Pelayo Fernández } \\
\text { Superfície }\end{array}$} & \multirow[b]{2}{*}{ Total } \\
\hline & $\begin{array}{l}\text { Coluvión Infe- } \\
\text { rior+Superior }\end{array}$ & $\begin{array}{l}\text { Coluvión Infe- } \\
\text { rior }\end{array}$ & Fluvial & Coluvial & & \\
\hline $\begin{array}{c}\text { Cantos/bloques } \\
\text { brutos }\end{array}$ & 102 & - & 92 & 130 & - & 324 \\
\hline $\begin{array}{c}\text { Elementos de } \\
\text { percusión }\end{array}$ & - & - & 6 & 15 & - & 21 \\
\hline $\begin{array}{l}\text { Lascas enteras y } \\
\text { fragmentos }\end{array}$ & 165 & 155 & 1107 & 831 & - & 2103 \\
\hline Restos de talla & - & - & 364 & 205 & - & 569 \\
\hline Núcleos & 105 & 21 & 91 & 117 & - & 313 \\
\hline Utensilios & 258 & 75 & 162 & 180 & - & 600 \\
\hline LCTs y frag. & 143 & 67 & 34 & 143 & 263 & 583 \\
\hline Total & 773 & 318 & 1856 & 1621 & 263 & 4513 \\
\hline
\end{tabular}

${ }^{1}$ Entre estas caben señalar los materiales de las excavaciones de E. Aguirre (Museo "Quiñones de León” de Vigo) y J. M. Vidal Encinas (Museo de Pontevedra) y las colecciones de materiales de superficie de J. M. Álvarez Blázquez (Museo “Quiñones de León” de Vigo) y Pelayo Fernández (colección particular). 


\subsection{Aspectos tafonómicos}

Solo respecto a las intervenciones de Vidal Encinas contamos con información cuantitativa acerca del estado de conservación de la industria de GB, datos que creemos que pueden ser extrapolables al resto de series conocidas a tenor de lo que hemos podido observar directamente al revisarlas (excavación de E. Aguirre y colecciones Álvarez Blázquez y Pelayo Fernández, principalmente). Los materiales de procedencia coluvial presentan signos netos de alteración mecánica por rodamiento en torno al $70 \%$ de los casos, por lo que el porcentaje de piezas con aristas en aparente buen estado es sensiblemente bajo (MÉNDEZ-QUINTAS, 2008). En el caso de los materiales de dinámicas sedimentarias fluviales el estado de conservación de las piezas es sensiblemente diferente, ya que el $76,6 \%$ presenta escasas o nulas huellas de desgaste erosivo.

El análisis de los tamaños de la industria revela diferencias notables entre las series de origen lateral y fluvial. En el primer grupo destacan los rangos de mayor tamaño, mientras que en el segundo se observa un predominio de la fracción menor (MÉNDEZ-QUINTAS, 2007, 2008). Esta situación pone de relieve importantes diferencias tafonómicas entre ambos, en principio indicativos de condiciones de integridad aceptables en los materiales de origen fluvial. Las referencias señaladas (CERQUEIRO LANDín, 1996) a orientaciones preferentes de las piezas en las excavaciones de D. Cerqueiro son interesantes por qué evidencian procesos tafonómicos fluviales; en las planimetrías publicadas de las excavaciones de E. Aguirre estas orientaciones son perfectamente visibles en los niveles de origen lateral (AGUIRRE, 1964). En todo caso es un aspecto que deberá ser contrastado en futuras intervenciones.

\subsection{Captación de materias primas}

De forma generalizada los tipos de rocas explotados en GB corresponden a dos grandes grupos: cuarzos y cuarcitas. Existen diferencias importantes en la captación de cada grupo que se manifiestan en su gestión. El cuarzo es de acceso inmediato en el yacimiento, ya que las terrazas del río Louro y los depósitos miocenos subyacentes contienen cuarzo en tamaños y morfologías apropiados para la talla. En un radio inferior a los $2 \mathrm{~km}$ existen múltiples lugares donde abastecerse de esta materia prima. La cuarcita, por su parte, no se observa en las cargas aluviales de la cuenca del río Louro, ya que en la región la única cuarcita existente es la que se encuentra en las formaciones del Miño. Como ya hemos comentado la cuenca del Louro fue capturada a lo largo del Pleistoceno medio por la del Miño, con anterioridad al emplaza- miento de las terrazas T4 $(+30-39 \mathrm{~m})$. En ese proceso el Miño depositó en su área de confluencia importantes niveles de arenas y gravas de cuarcita que aún son visibles a escasos $1,5 \mathrm{~km}$ de la sección clásica de GB. Ahora bien, el tamaño y el grado de alteración de estos cantos invita a pensar que esta no fue la zona preferente de obtención. La fuente más probable estaría, cuando menos, a unos $3 \mathrm{~km}$ del yacimiento, en las formaciones del Miño más próximas, en especial en la propia llanura de inundación de este río de la terraza T3, coetánea del yacimiento, con abundantísimos cantos de cuarcita de tamaños y morfologías adecuadas para la talla.

La representación de ambas materias primas varía de forma notable entre las series, sobre todo entre las colecciones de superficie y las series líticas producto de excavación. Entre las primeras se observa un predominio marcado de elementos de cuarcita, circunstancia derivada de su mayor facilidad de identificación frente a las piezas realizadas en cuarzo. Entre los segundos la distribución entre ambas rocas es más proporcionada, con un ligero predominio del cuarzo frente a la cuarcita $-57,4 \%$ y $42,6 \%$, respectivamente, entre los materiales de procedencia fluvial (MÉNDEZ-QUINTAS, 2007).

\subsection{Estructura tecnoeconómica de la industria lítica. Aspectos generales}

En la imagen que se desprende del análisis de las diferentes fases de las cadenas operativas, sobre todo en el caso de las series más representativas, como en los materiales de origen fluvial de las excavaciones de J. Vidal Encinas, destaca el predominio de los elementos propios de la fase de producción. La siguiente fase en importancia es la del consumo, que gana en significación si se evalúa en función del peso. Los elementos asociados a la adquisición no son demasiado relevantes ni en número de efectivos, ni en peso, circunstancia relacionable en especial con las pautas de aprovisionamiento de las materias primas ya mencionadas.

En base a los datos anteriores es posible reconocer una verdadera economía en la gestión de la cuarcita y el cuarzo. La cuarcita aparece en el yacimiento en fase de producción y consumo, se introducen núcleos ya testados y en fase de explotación, así como grandes lascas y LCTs ya configurados. El cuarzo, adquirido en su mayor parte en el yacimiento o en su entorno próximo, aportó un importante volumen de productos, mayor que el de la cuarcita, aunque esta última roca fue elegida de manera preferente para la configuración de utensilios.

Este comportamiento económico y tecnológico deriva sin duda de las condiciones de accesibilidad de las diferentes materias primas y de las actividades desarrolladas en el yacimiento. Pero también tendría influencia la desigual respuesta de las materias primas explotadas. La cuarcita se presen- 
Eduardo Méndez-Quintas, Manuel Santonja, Alfredo Pérez-González, Lee J. Arnold, João Pedro Cunha Ribeiro, Martina Demuro, Mikel Díaz-Rodríguez, Mathieu Duval, Alberto Gomes, José Meireles, Sérgio Monteiro-Rodrigues \& Andrea Serodio Domínguez

taba en soportes con tamaño y formato muy aptos para la talla, y ofrecía una controlable respuesta a la percusión, permitiendo la fácil configuración de LCTs y una explotación más recurrente e intensa de los soportes. El cuarzo era una materia prima más "barata", de acceso inmediato, pero con una respuesta a la talla menos predecible que la cuarcita, aunque puede proporcionar filos con condiciones mecánicas similares o superiores a la cuarcita. Por esta razón el cuarzo pudo ser elegido para la obtención sistemática de lascas de filo cortante que solo en casos puntuales se transformaron mediante retoque en utensilios de morfología específica.

\subsection{Sistemas de explotación de los núcleos y productos}

Los sistemas de explotación identificados en el yacimiento están dominados por esquemas elementales, monopolares simples y periféricos (Fig. 10. 1-3). Junto a ellos cabe destacar el porcentaje de núcleos discoides y multipolares (Fig. 10. 4-8), estos últimos muy ligados a los soportes en cuarzo (ECHAIDE, 1971; MÉNDEZ-QUINTAS, 2007, 2008). También muy vinculado al cuarzo se encuentran ejemplos de núcleos explotados por talla bipolar sobre yunque (LOMBERA HERMIDA et al., 2011). En la intensidad de explotación se constatan diferencias notables. En primer lugar, en relación con las materias primas se observa que los núcleos en cuarcita son los que muestran mayor número de negativos. El análisis del grado de exhaustividad, en función del volumen residual sin explotar de los núcleos, permite observar que en algunos casos se conservan volúmenes susceptibles de seguir produciendo soportes, pero una gran parte de los núcleos están agotados o con un aprovechamiento muy intenso. En contadas ocasiones se observan núcleos en una fase inicial de explotación.

Los productos obtenidos son esencialmente lascas, cuya variabilidad tecnotipológica y grado de corticalidad invitan a pensar en una producción esencialmente realizada en el propio yacimiento. En general se observa el marcado predominio de lascas ordinarias o con escasos restos corticales, si bien también es significativa la presencia de otras enteramente corticales, válidas para demostrar que el inicio de la explotación de una parte de los soportes se ha efectuado en el propio yacimiento. En el caso de la cuarcita habría que matizar que los materiales configurados parece que se introdujeron ya acabados en el yacimiento.

La presencia de lascas con dorso natural, fácilmente obtenibles a partir de cantos rodados, resulta altamente significativa. Otros tipos de lascas son poco frecuentes, por ejemplo, las lascas kombewa, o productos específicos de los esquemas de explotación discoidal, tales como lascas desbordantes o puntas pseudolevallois. De manera testi- monial se puede señalar alguna lasca de morfología levallois, si bien pueden proceder de la explotación de núcleos discoides o incluso de la configuración/ reavivado de algún LCT, ya que no se han registrado núcleos explotados con arreglo estricto a dicho concepto.

\subsection{Utensilios retocados}

En todas las colecciones analizadas se reconocen utensilios retocados en porcentajes comprendidos entre el $6 \%$ y el $15 \%$ del total de efectivos. Entre los tipos más habituales están los denticulados y las raederas, con predominio de los primeros. También se tiene constancia de cierto número de perforadores, becs y lascas con retoques aislados. En número mucho más limitado se cuentan algunos cuchillos de dorso, raspadores y esporádicamente alguna pieza afín a buril. Los cantos tallados también se mencionan en las colecciones estudiadas, pero nuestras revisiones invitan a limitar su importancia numérica. A nivel de materias primas se hace evidente la predilección por la cuarcita frente al cuarzo, el uso generalizado de lascas parcial o enteramente corticales y la tendencia a elegir los soportes de mayores dimensiones.

El retoque aplicado en la conformación de este tipo de piezas se caracteriza por una extensión marginal, inclinación simple y modo directo. Esta norma general se mantiene sin gran desviación en los distintos tipos de materia prima. En consecuencia, la aplicación del retoque en GB modificó escasamente el aspecto general de los soportes, dando lugar a la inexistencia de patrones morfológicos en la delineación de los bordes.

\subsection{Large cuttings tools (LCTs)}

Los LCTs son especialmente abundantes en todas las series analizadas. En algunas series llegan a destacar los hendedores sobre los bifaces, y también se observa una buena representación de triedros.

Los bifaces, salvo excepciones, responden a la idea de bifaces-útil y raramente se identifican ejemplos de bifaces soporte de útil (BOËDA, 2001); como soportes se han utilizado tanto lascas como cantos rodados y responden a morfologías apuntadas -amigdaloides y lanceoladas preferentemente(Fig. 11. 1-4 y 6) o de filo transversal (Fig. 11. 5 y 7). En general presentan morfologías equilibradas en ambos planos de simetría, una intensidad de reducción amplia y eventualmente se constata la utilización de percutores elásticos en regularización y finalizado.

La presencia de hendedores en GB resulta realmente notable (VIDAL ENCINAS, 1983; MÉNDEZ-QUINTAS, 2007). Se acomodan bien a los tipos definidos por Tixier (1956), esencialmente de 

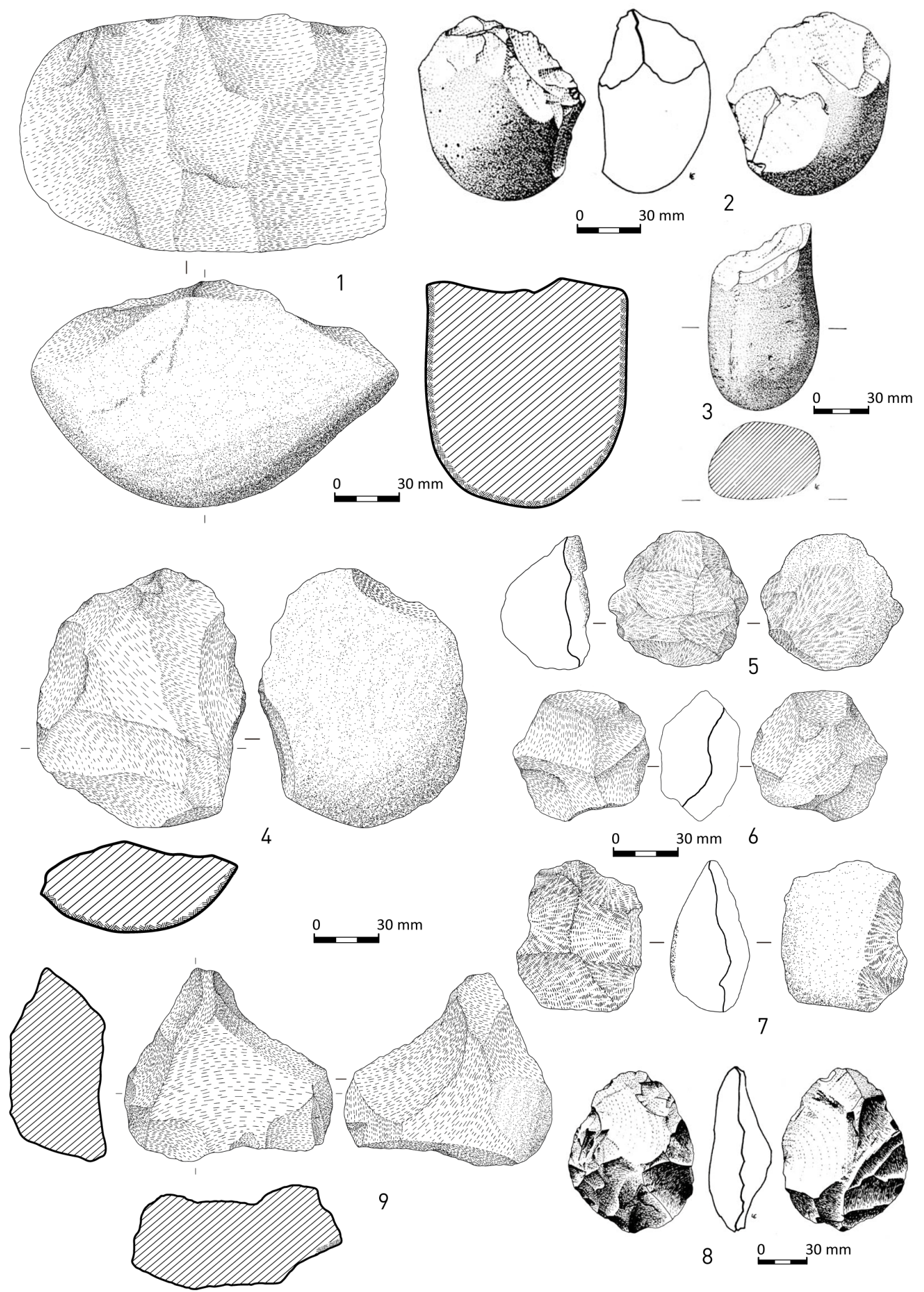

Fig. 10. Ejemplos de diferentes tipos de núcleos. 1 y 3: monopolares, 2: periférico, 4-8: discoides y 9: morfología levallois. Todos en cuarcita salvo 5 y 7 en cuarzo. Dibujos 2, 3 y 8 (ECHAIDE 1971).

Fig. 10. Exemples of different types of cores. 1 and 3: monopolar, 2. peripheral, 4-8: discoidal and 9. levallois morphology. All made of quartzite except 5 and 7, made of quartz. Drawings 2, 3 and 8 (ECHAIDE 1971). 
Eduardo Méndez-Quintas, Manuel Santonja, Alfredo Pérez-González, Lee J. Arnold, João Pedro Cunha Ribeiro, Martina Demuro, Mikel Díaz-Rodríguez, Mathieu Duval, Alberto Gomes, José Meireles, Sérgio Monteiro-Rodrigues \& Andrea Serodio Domínguez
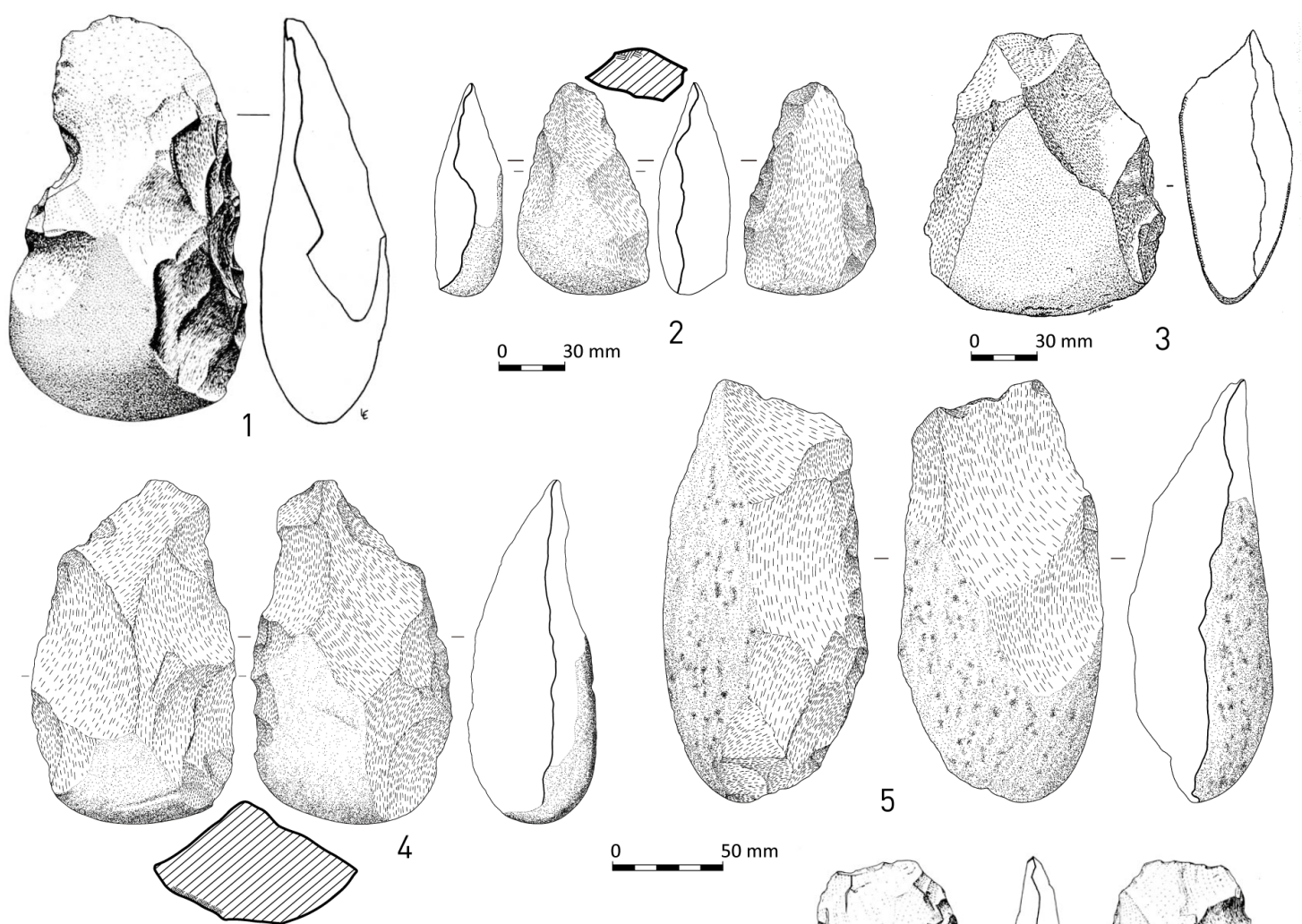

2
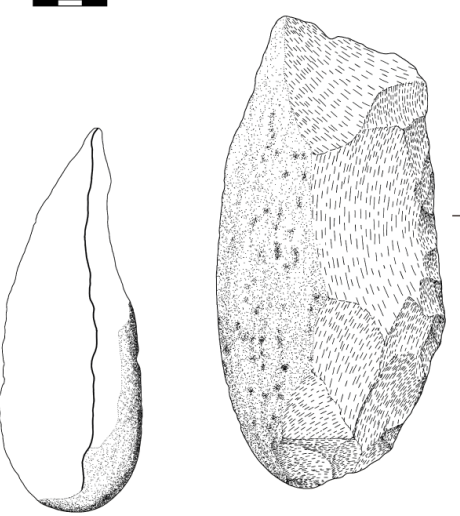

5
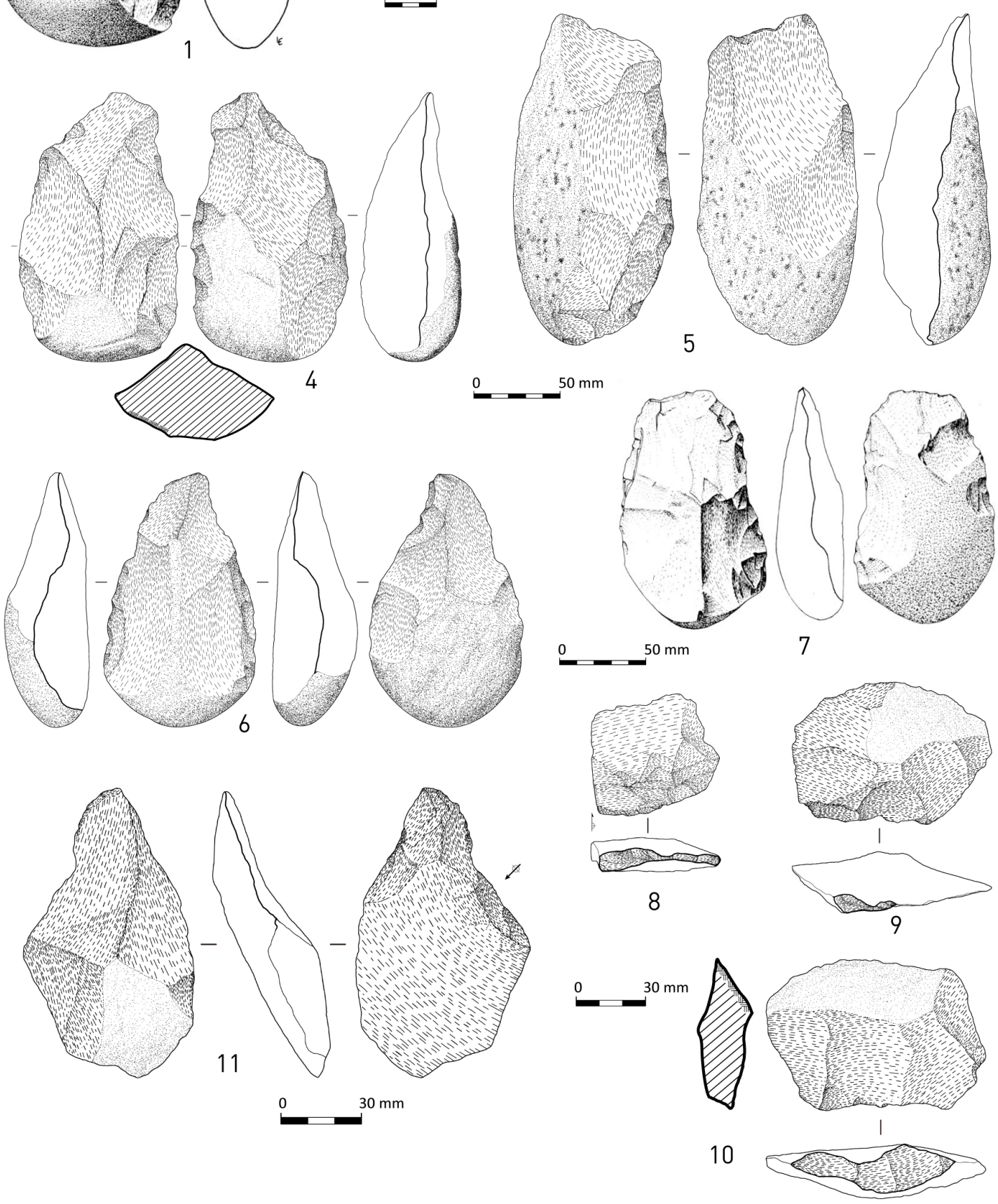

Fig. 11. Bifaces con diferentes morfologías (1-7) y productos de configuración/mantenimiento de LCTs (8-11), todos de cuarcita. Dibujo 1 y 7 (ECHAIDE, 1971); 3 (VIDAL ENCINAS, 1982a).

Fig. 11. Bifaces of different morphologies (1-7) and products of shaping/maintenance of LCTs (8-11), all made of quartzite. Drawings 1 and 7 (ECHAide 1971); 3 (VIDAL ENCINAS 1982a). 
tipo O (Fig. 12. 1-2), aunque también se identifican los tecnotipos I y II (Fig. 12. 3-5) e incluso algunos sobre lasca kombewa -tipo VI- (Fig. 12.6-7). Muestran densidad dispar en la intensidad del retoque, en ocasiones con percutor elástico, pero al margen de esto mantienen un acentuado grado de simetría, derivado con frecuencia de la morfología inicial de la lasca soporte.

Los triedros participan de los mismos principios de configuración que bifaces y hendedores, representados por piezas con extremidades apuntadas bien despejadas y buena simetría frontal y bilateral, conseguidas mediante series largas de extracciones, a veces mediante el uso de percutores elásticos (Fig. 13).

En las series procedentes de excavación hemos llegado a observar porcentajes relevantes de subproductos de configuración y mantenimiento de LCTs, especialmente lascas o extremos apicales (Fig. 11. 8-11). La presencia de estos elementos indica que este tipo de tareas se realizó en el propio yacimiento, especialmente las de mantenimiento, ya que en general parece que estos utensilios fueron introducidos ya configurados en el yacimiento.

\section{EL YACIMIENTO DE GÁNDARAS DE BUDIÑO EN EL CONTEXTO DEL ACHE- LENSE IBÉRICO}

A falta de realizar nuevos trabajos de investigación, los datos actuales no dejan lugar a muchas dudas acerca del marco cronológico general y de las principales características tecnotipológicas de la industria de Budiño. Estamos ante un yacimiento plenamente achelense cuya cronología se puede situar tentativamente en la segunda mitad del Pleistoceno Medio, similar a la de otros yacimientos achelenses también en la cuenca del río Miño (MENDEZ-Quintas et al., 2018).

\subsection{Marco y desarrollo cronológico del ache- lense peninsular}

El primer poblamiento humano de la Península Ibérica se desarrolla con anterioridad al millón de años, en el mismo contexto que el resto del mediodía europeo. Yacimientos como los de Barranco León y Fuente Nueva 3 (cuenca de Guadix-Baza, Granada), los niveles inferiores de Gran Dolina y Sima del Elefante en la Sierra de Atapuerca (Burgos) son ejemplos consistentes de presencia humana (en forma de industria lítica y/o restos paleoantropológicos) en rangos cronológicos comprendidos entre 1,4-0,8 m. a. (TORO-MOYANO et al., 2011; Duval et al., 2014; MORENO et al., 2015; PARÉs et al., 2018).

Al margen de estos primeros episodios de presencia humana con industrias de núcleos y las- cas no achelenses, algunos investigadores consideran que la primera presencia achelense a nivel peninsular la tendríamos en el yacimiento de La Boella (Tarragona), el cual se ha datado por paleomagnetismo y cosmogénicos entre 1,0 y $0,8 \mathrm{~m}$.a (VALlVERDU et al., 2014; MOSQUERA et al., 2015). Ahora bien, las características de los materiales recuperados (serie limitada numéricamente, compuesta por lascas, restos de talla, núcleos y algunos configurados) no permiten una inequívoca atribución a este tecnocomplejo (SANTONJA et al., 2016a; MENDEZ-QuinTAS et al., 2018). Otra referencia que a veces se menciona es la del yacimiento de Cueva Negra del Estrecho del Río Quipar Murcia- (SCOTT \& GIBERT, 2009; WALKER et al., 2013), si bien con los datos publicados tampoco es posible asumir la identidad achelense de este yacimiento (JIMÉNEZ-ARENAS et al., 2011) En cronologías próximas al límite Matuyama/Brunhes hay que mencionar también algunas localizaciones de industria lítica asociados a terrazas fluviales con cotas superiores a $+40 \mathrm{~m}$ en la Meseta (SANTONJA Y PÉREZ-GONZÁLEZ 2010), los más destacados los reconocidos en el tramo medio del valle del Tajo (RUBIO-JARA et al., 2016).

La verdadera eclosión achelense en la Península Ibérica se desarrolla con posterioridad al MIS 13 extendiéndose hasta el MIS 6. En este lapso temporal de unos $400 \mathrm{ka}$ el número de yacimientos constatados se multiplica de forma exponencial. $\mathrm{La}$ mayor parte de ellos se asocian con las terrazas medias de las grandes cuencas atlánticas, con cotas comprendidas entre $+40 \mathrm{~m} \mathrm{y}+20 \mathrm{~m}$ (TEXIER et al., 1995; SANTONJA \& VILLA, 2006; SANTONJA \& PÉREZ-GONZÁLEZ, 2010; RUBIO-JARA et al., 2016; SANTONJA et al., 2016a; CUNHA et al., 2017). La extensión de programas de datación numérica para los sistemas fluviales de las cuencas ibéricas confirma para el achelense peninsular este rango cronológico (MARTINS et al., 2010a; MARTINS et al., 2010b; MORENO et al., 2012; Rosina et al., 2014; ProençA CUNHA et al., 2016; MORENO et al., in press; SiLva et al., 2017) en el que encaja perfectamente GB. La posición de Budiño en relación con una terraza de $+23 \mathrm{~m}$ constituye un indicador fiable para atribuirle una cronología dentro de la segunda mitad del Pleistoceno Medio, en el margen temporal que se contempla para otros yacimientos achelense regionales, caso del yacimiento de Porto Maior sobre una terraza de $+34 \mathrm{~m}$, con fechas del MIS 8-9 (MENDEZ-QUINTAS et al., 2018).

\subsection{Principales características tecnológicas del achelense ibérico}

Las características tecnomorfológicas de la industria de GB se inscriben de lleno en el Achelense de grandes lascas (LFA lato sensu Sharon, 2010) de facies africana reconocido en el resto de 
Eduardo Méndez-Quintas, Manuel Santonja, Alfredo Pérez-González, Lee J. Arnold, João Pedro Cunha Ribeiro, Martina Demuro, Mikel Díaz-Rodríguez, Mathieu Duval, Alberto Gomes, José Meireles, Sérgio Monteiro-Rodrigues \& Andrea Serodio Domínguez
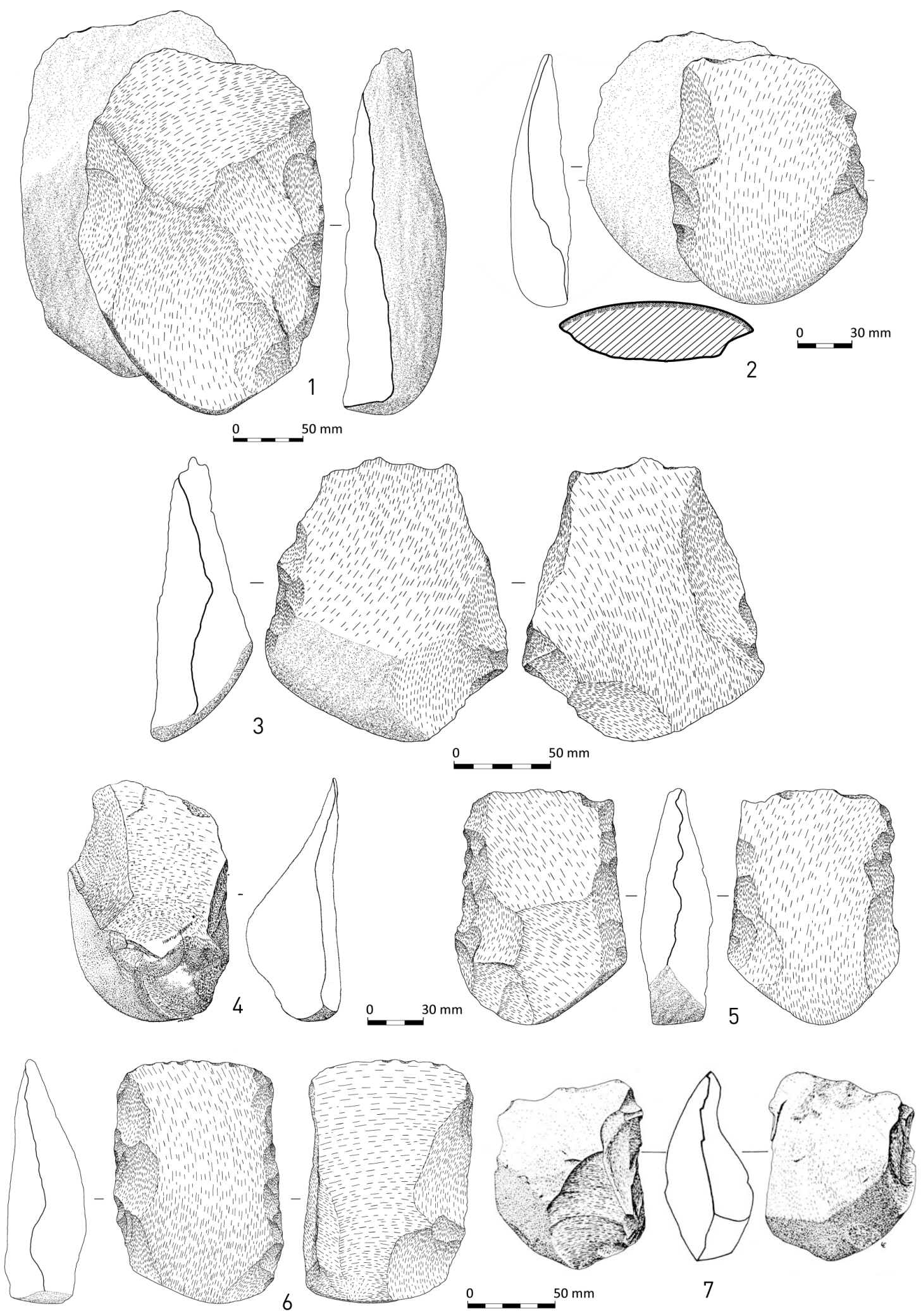

Fig. 12. Hendedores en cuarcita. 1-2: tipo O, 3-5: tipo II y 6-7: tipo VI. Figura 4 (VIDAL EnCINAS 1982a) y 7 (ECHAIDE 1971 ). Fig. 12. Cleavers made of quartzite. 1-2: type 0, 3-5: type II and 6-7: type VI. Figure 4 (VIDAL ENCINAS 1982a) and 7 (ECHAIDE 1971). 

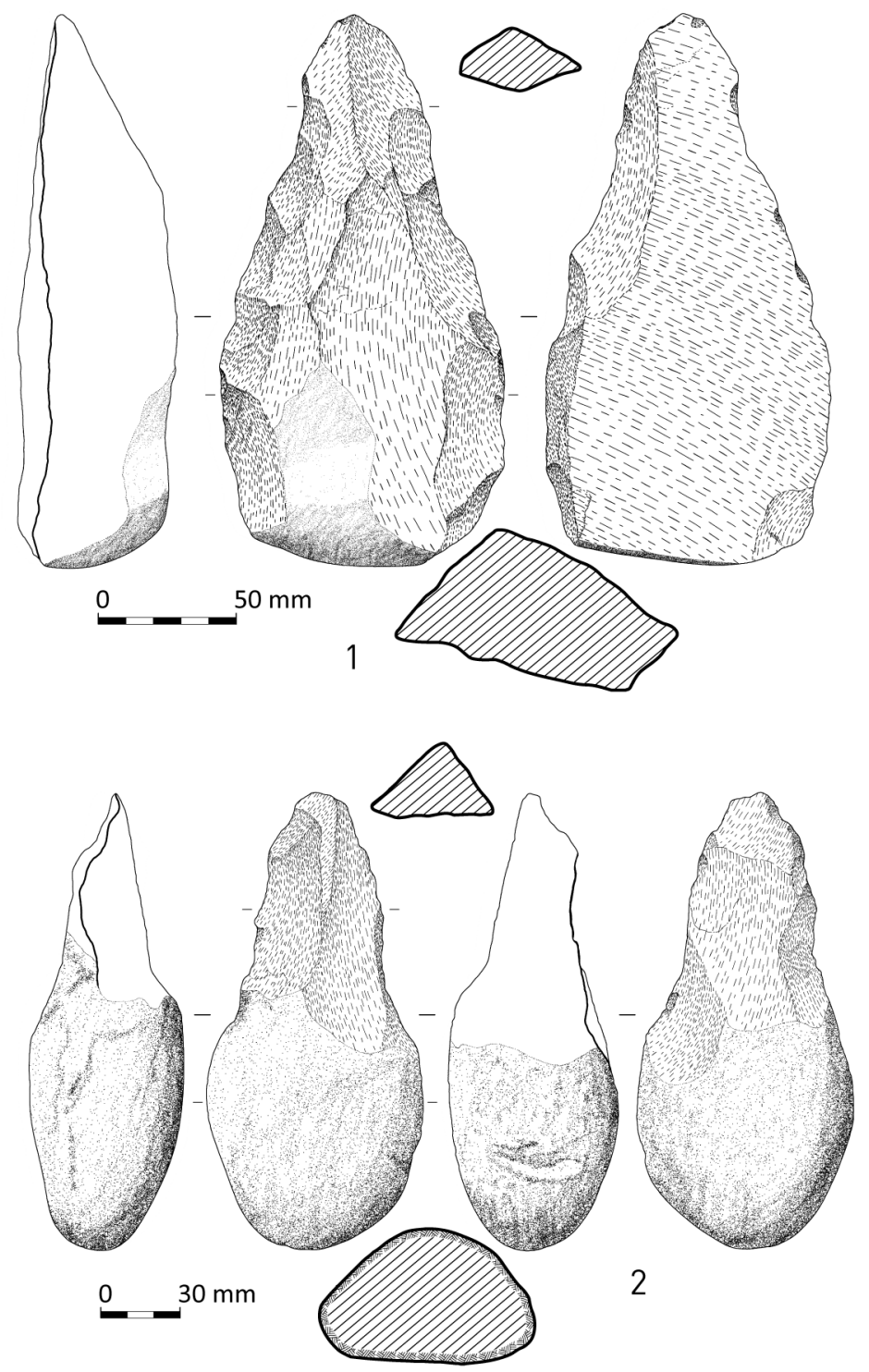

Fig. 13. Triedros sobre lasca (1) y canto (2) de cuarcita.

Fig. 13. Trihedral picks on flake (1) and on pebble (2) made of quartzite.

la fachada atlántica de la Península Ibérica (MeIreles, 1992; CUNHA Ribeiro, 1999; SANTONJA \& VILLA, 2006; SANTONJA \& PÉREZGONZÁLEZ, 2010; SANTONJA et al., 2016a; SHARON \& BARSKY, 2016; MENDEZ-QUINTAS et al., 2018). Igual que en Budiño, el achelense peninsular se caracteriza tecnológicamente por la gestión y consumo de grandes volúmenes de materias primas de cara a la obtención de grandes lascas soporte. Estas lascas pueden ser utilizadas directamente o bien transformadas en LCTs (bifaces, hendedores, triedros, macroraederas, etc.). Asociados a estas cadenas operativas de configuración o "façonnage", las cadenas operativas de producción o "debitage" (BÖEDA et al., 1990) pueden alcanzar una importancia paralela. Estos procesos aportaban lascas de pequeño o mediano formato y filo cortante, obtenidas mediante sistemas de explotación sencillos (monopolares longitudinales, multipolares y discoides) y podían ser transformadas posteriormente por retoque en utensilios más complejos. A partir de estos principios básicos que definen la esencia del Achelense hay que tener en cuenta que el predominio de una fase u otra de la cadena operativa, o la presencia mayor o menor de LCTs en un yacimiento, incluso su ausencia, viene determinada por factores múltiples de índole funcional, sedimentario, de la disponibilidad de materias primas o de otras variables difíciles de identificar en la actualidad (SANTONJA \& QUEROL, 1980; POTTS 
Eduardo Méndez-Quintas, Manuel Santonja, Alfredo Pérez-González, Lee J. Arnold, João Pedro Cunha Ribeiro, Martina Demuro, Mikel Díaz-Rodríguez, Mathieu Duval, Alberto Gomes, José Meireles, Sérgio Monteiro-Rodrigues \& Andrea Serodio Dominguez

et al., 1999; DELAGNES et al., 2006; GOREN-INBAR \& SHARON, 2006; DOMINGUEZ- RODRIGO et al., 2009; RUBIO-JARA et al., 2017). La representatividad de las diferentes fases de la cadena operativa (GENESTE, 1991) puede variar notablemente, reconociéndose incluso escenarios en que predominan los elementos asociados a las fases de producción (lascas, núcleos y restos de talla), el yacimiento de Áridos-1 (SANTONJA \& QUEROL 1980), sin ningún LCT constituye un buen ejemplo de una situación de este tipo.

Otra característica del Achelense ibérico, compartida por Budiño, consiste en el uso mayoritario de materias primas de carácter local, es decir accesibles en un reducido radio desde el yacimiento -generalmente inferior a los $5 \mathrm{~km}$ - (SANTONJA \& VILla, 1990; MEIRELES \& CUNHA RIBEIRO, 19911992; SANTONJA, 1992, 1996). En ocasiones se identifican desplazamientos de materias primas a mayores distancias. El ejemplo más notable es el de los yacimientos de Ambrona y Torralba, en los que se introdujeron variedades de sílex desde más de $30 \mathrm{~km}$ de distancia (SANTONJA et al., 2016b). Otra situación interesante es la que se produce en el paleolítico del Manzanares (Madrid), donde se observa la importación sistemática de cuarcita procedente del valle del Jarama, con distancias que pueden llegar a superar los $10 \mathrm{~km}$ (SANTONJA, 1996; RUBIO-JARA et al., 2016). En lo que respecta al uso de diferentes tipos de rocas accesibles, se observa claramente una economía de materias primas (Perles, 1991), al consumirlas de manera diferenciada en función de sus propiedades mecánicas, tamaño y las estrategias de explotación y/o configuración. Con frecuencia las variantes más aptas para la talla experimentan una intensificación en su explotación, hasta el agotamiento del soporte en el caso de los núcleos, o experimentando un afilado y reacondicionamiento sistemático en el caso de las piezas configuradas (SANTONJA \& VILLA, 2006).

Dadas las condiciones geológicas de la Península Ibérica -el macizo Hespérico ocupa más del $60 \%$ de la superficie emergida de la Península y se caracteriza por la abundancia de materiales metamórficos- las cuarcitas han sido las rocas más empleadas. Con frecuencia suponen más del $80 \%$ de la materia prima en los yacimientos asociados a dominios fluviales, aun cuando en el entorno existan otras con condiciones aptas para la talla. El cuarzo, habitualmente asociado a la cuarcita, también fue un recurso habitual, si bien en porcentajes que no suelen exceder el 20\% (SANTONJA, 1992, 1996). El sílex, cuya distribución en la Península es más limitada que la de la cuarcita, se utilizó de manera normalizada donde es fácilmente accesible, caso de la vertiente mediterránea y zonas restringidas del interior y el occidente ibérico, caso por ejemplo del valle del Mazanares (SANTONJA \& VILLA, 2006; OLLÉ et al., 2013; RUBIO-JARA et al.,
2016). A pesar de sus innegables cualidades mecánicas, cuando está asociada a otras, por ejemplo, la cuarcita, se detecta un uso diferencial, escogiendo sílex para la obtención de lascas brutas, fueran o no retocadas, y con frecuencia prefiriendo cuarcita para los LCTs, en especial para los hendedores (SANTONJA \& Villa, 2006; Ollé et al., 2013; RUBIO-JARA et al., 2016). La utilización de otras rocas (caliza, arenisca, lidita, jaspe, basalto, riolita...) es muy esporádica, condicionado a las condiciones litológicas del entorno del yacimiento.

\section{CONCLUSIONES}

A pesar de que han transcurrido más de 50 años desde el descubrimiento del yacimiento de As Gándaras de Budiño, el debate en torno a él sigue sin estar totalmente cerrado. Con los datos actuales, y a la espera de los resultados de futuras investigaciones, se puede definir a Budiño como una estación que encaja plenamente en el achelense de grandes lascas de raíz africana. Totalmente comparable desde el punto de vista tecnológico y del uso o gestión de materias primas al resto de enclaves achelenses de la Península Ibérica. Los mayores problemas que subsisten para avanzar en la interpretación paleoambiental y funcional del yacimiento se refieren a la indefinición estratigráfica que subsiste a pesar de todas las investigaciones desarrolladas en el yacimiento. Por el contrario, no existen dificultades metodológicas o de otro tipo en relación con el análisis de los materiales líticos.

Al yacimiento, asociado a las facies fluviales de la terraza de $+23 \mathrm{~m}$ del río Louro, le correspondería una cronología dentro de la segunda mitad del Pleistoceno Medio. Los datos que se manejan actualmente para la cuenca del Miño dibujan una presencia importante de grupos humanos con tecnología achelense en la zona entre el MIS 9 y 6 , desde c. 350 a $150 \mathrm{ka}$, rango cronológico en el que se situaría Budiño. Los niveles laterales que en su día fueron fechados por ${ }^{14} \mathrm{C}$, con dataciones que remitían a momentos del último máximo glaciar, podrían ser considerados en el mejor de los casos como edades mínimas, o simplemente deberían dejar de tomarse en consideración. Estas edades señalarían en todo caso una fase de movimiento y redeposición de la industria, pero sin ninguna relación con la etapa de ocupación humana que originó el yacimiento.

\section{AGRADECIMIENTOS}

Agradecer los comentarios a los revisores del artículo por todos sus comentarios, que han permitido mejorar notablemente el texto de este artículo. El trabajo de investigación desarrollado por Mathieu Duval está actualmente financiado por una Future Fellowship (FT150100215) del Australian Research Council (ARC). 


\section{BIBLIOGRAFÍA}

AguirRe, E., 1964. Las Gándaras de Budiño Porriño (Pontevedra). Excavaciones Arqueologicas en España 31: 28.

Aguirre, E., Butzer, K.W., 1967. Problematical Pleistocene artifact assemblage from Northwestern Spain. Science 157 (3787): 430-431.

Bö̈DA, E., 2001. Determination des unités tecnofonctinelles de pièces bifaciales provenant de la couche acheuléenne C'3 Base du site de Barbas I, en: Cliquet, D. (Ed.), Les industries à outils bifaciaux du Paléolithique moyen d'Europe occidentale. ERAUL 98: 51-75.

BöedA, E., Geneste, J.M., Meignen, L., 1990. Identification de chaînes opératoires lithiques du Paléolithique ancien et moyen. Paleo 2 (1): 43-80.

Butzer, K.W., 1967. Geomorphology and Stratigraphy of the Palaeolithic site of Budiño. Eiszeitalter und Gegenwart 18 (31-32): 82-103.

CANO PAN, J.A., 1993. Análisis historiográfico del Paleolítico en Galicia. Semata 5: 29-52.

Cerqueiro Landín, D., 1996. As Gándaras de Budiño: Prehistoria e Historia, en: Fábregas Valcarce, R. (Ed.), Os Primeiros Poboadores de Galicia: O Paleolítico. Cadernos do Seminario de Sargadelos 73.

Cunha, P.P., Martins, A.A., Buylaert, J.-P., Murray, A.S., Raposo, L., Mozzi, P., Stokes, M., 2017. New data on the chronology of the Vale do Forno sedimentary sequence (Lower Tejo River terrace staircase) and its relevance as a fluvial archive of the Middle Pleistocene in western Iberia. Quaternary Science Reviews 166: 204-226. 10.1016/ j.quascirev.2016.11.001

CunHA RIBEIRO, J.-P., 1999. O acheulense no centro de Portugal: o Vale do Lis. Contribuicão para uma abordagem tecno-tipológica das suas indústrias líticas e problemática do seu contexto cronoestratigráfico. Facultade de Letras, Universidade de Lisboa.

Cunha Ribeiro, J.-P., Monteiro-Rodrigues, S., Gomes, A., Méndez Quintas, E., Meireles, J., PérezGonZÁlez, A., SANTONJA, M., 2017. Ocupações Pleistocénicas na margem esquerda do Baixo Minho (Miño/Minho 2). Objetivos e primeiros resultados de um projeto transfronteiriço, en: VV.AA (Ed.), Arqueologia em Portugal / $2017-$ Estado da Questão. Associação dos Arqueólogos Portugueses: 303-318.

De Vicente, G., Cloetingh, S., Van Wees, J.D., Cunha, P.P., 2011. Tectonic classification of Cenozoic Iberian foreland basins. Tectonophysics 502: 38-61. 10.1016/j.tecto.2011.02.007

De Vicente, G., Vegas, R., 2009. Large-scale distributed deformation controlled topography along the western Africa-Eurasia limit: Tectonic constraints. Tectonophysics 474: 124-143. 10.1016/ j.tecto.2008.11.026

Delagnes, A., Lenoble, A., Harmand, S., Brugal, J.-P., Prat, S., Tiercelin, J.-J., Roche, H., 2006. Interpreting pachyderm single carcass sites in the African Lower and Early Middle Pleistocene record: A multidisciplinary approach to the site of Nadung'a 4
(Kenya). Journal of Anthropological Archaeology 25 (4): 448-465.

Domínguez- Rodrigo, M., Alcala, L., LuQue, L., 2009. Peninj. A Research Project on Human Origins 1995 -2005 , en: Book, O. (Ed.), American School of Prehistoric Research Monograph Series.

Duval, M., Bahain, J.-J., Falguères, C., Garcia, J., Guilarte, V., Grün, R., Martínez, K., Moreno, D., ShaO, Q., Voinchet, P., 2014. Revisiting the ESR chronology of the Early Pleistocene hominin occupation at Vallparadís (Barcelona, Spain). Quaternary International. 10.1016/j.quaint.2014.08.054

ECHAIDE, M.D., 1971. La industria lítica del yacimiento de Budiño (Pontevedra, España). Munibe XXIII (1): 125-154.

FreEMAN, L.G., 1976. Acheulean sites an stratigraphy in Iberia and the Maghreb, en: Butzer, K.W., Isaac, G.L. (Eds.), After the Australopithecines : stratigraphy, ecology, and culture, change in the Middle Pleistocene. World anthropology: 661-743.

GENESTE, J.M., 1991. L'approvisionnement en matieres premieres dans les systemes de production lithique: la dimension spatiale de la technologie Treballs d'Arqueologia 1: 1-36.

Goren-InBAR, N., SHARON , G., 2006. Invisible Handaxes and Visible Acheulian Biface Technology at Gesher Benot Ya'aqov, Israel, en: Goren-Inbar, N. Sharon, G. (Eds.), Axe Age: Acheulian Toolmaking from Quarry to Discard: 111-135.

Gracia Prieto, F.J., Cano Pan, J., Giles Pacheco, F., Santiago, A., Mata Almonte, E., Gutiérrez, J.M., 2004. Evolución geomorfológica de la cuenca del río Louro en conexión con el valle del Miño y poblamiento paleolítico (Gándaras de Budiño-Tuy; Pontevedra). Zona Arqueológica 4 (IV): 218-229.

Howell, F.C., Butzer, K.W., Freeman, L.G., Klein, R.G., 1995. Observations on the Acheulean occupation site of Ambrona (Soria Province, Spain), with particular reference to recent investigation (19801983) and the lower occupation. Jahrbuch des Römisch-Germanischen Zentralmuseum Mainz 38: 33-82.

JimÉneZ-Arenas, J.M., SANTONJA, M., Botella, M., PALMQVIST, P., 2011. The oldest handaxes in Europe: fact or artefact? Journal of Archaeological Science 38 (12): 3340-3349. 10.1016/ j.jas.2011.07.020

JordÁ CERdÁ, F., 1986. Paleolítico, en: Montendgro Duque, A. (Ed.), Historia de España. Prehistoria. Editorial Gredos: 7-150.

Lombera Hermida, A., Rodríguez, X.-P., FÁbregas, R., MONCEL, M.-H., 2011. La gestion du quartz au Pléistocène moyen et supérieur. Trois exemples d'Europe Méridionale. L'Anthropologie 115 (2): 294-331. 10.1016/j.anthro.2011.02.003

Martín-Serrano, A., Molina, E., 2005. El Macizo Ibérico, en: IGME (Ed.), Mapa Geomorfológico de España y del margen continental a escala 1:1.000.000. Memoria: 65-85.

Martins, A.A., Cunha, P.P., Buylaert, J.-P., HuOt, S. Murray, A.S., Dinis, P., StOKES, M., 2010a. KFeldspar IRSL dating of a Pleistocene river terrace staircase sequence of the Lower Tejo River (Portugal, western Iberia). Quaternary Geochronology 5 (2-3): 176-180. 
Eduardo Méndez-Quintas, Manuel Santonja, Alfredo Pérez-González, Lee J. Arnold, João Pedro Cunha Ribeiro, Martina Demuro, Mikel Díaz-Rodríguez, Mathieu Duval, Alberto Gomes, José Meireles, Sérgio Monteiro-Rodrigues \& Andrea Serodio Domínguez

Martins, A.A., Cunha, P.P., Rosina, P., Osterbeek, L., Cura, S., Grimaldi, S., Gomes, J., Buylaert, J.P., Murray, A.S., Matos, J., 2010b. Geoarchaeology of Pleistocene open-air sites in the Vila Nova da Barquinha-Santa Cita area (Lower Tejo River basin, central Portugal). Proceedings of the Geologists' Association 121 (2): 128-140.

MeIRELES, J., 1992. As industrias liticas do litoral minhoto. Contexto cronoestratigrafico e paleoambiental. Cadernos de Arqueologia Monografias 7: 577.

Meireles, J., CunHA Ribeiro, J.-P., 1991-1992. Matériasprimas e indústrias líticas do Paleolítico Inferior Português representatividade e significado. Cadernos de Arqueologia 8-9: 31-42.

MÉnDEZ-QuinTAS, E., 2007. El yacimiento achelense de As Gándaras de Budiño. La industria en facies fluviales. Complutum 18: 27-45.

MÉNDEZ-QuinTAS, E., 2008. La industria lítica de las facies coluviales del yacimiento achelense de As Gándaras de Budiño. El Locus $\mathrm{V}$ en las excavaciones de Vidal Encinas. Zephyrus LXIII (2): 41-61.

MÉndez Quintas, E., SAntonja, M., PÉrez-GonzÁlez, A., Demuro, M., Duval, M., Arnold, L., 2017. O sistema de terraços da bacia baixa do rio Minho (margem galega), en: Gomes, A., Teixeira, J., Soares, L. (Eds.), $8^{\circ}$ Congreso Nacional de Geomorfología. Geomorfología 2017. Associação Portuguesa de Geomorfólogos, Facultade de Letras de Porto, pp. 57-58.

Mendez-Quintas, E., Santonja, M., Perez-Gonzalez, A., Duval, M., Demuro, M., ARnOld, L.J., 2018. First evidence of an extensive Acheulean large cutting tool accumulation in Europe from Porto Maior (Galicia, Spain). Scientific Reports 8 (1): 3082. 10.1038/s41598-018-21320-1

Miall, A.D., 1996. The Geology of Fluvial Deposits. Sedimentary Facies, Basin Analysis, and Petroleum Geology. Springer.

Moreno, D., Duval, M., Rubio-Jara, S., PANera, J., BAhain, J.J., Shao, Q., PÉreZ-GonzÁlez, A., FALGUÈRES, C., in press. ESR dating of Middle Pleistocene archaeo-paleontological sites from the Manzanares and Jarama river valleys (Madrid basin, Spain). Quaternary International. 10.1016/ j.quaint.2017.09.003

Moreno, D., Falguères, C., PÉrez-GonzÁlez, A., Duval, M., Voinchet, P., Benito-Calvo, A., Ortega, A.I., Bahain, J.-J., Sala, R., CarboNEll, E., BermúdeZ DE CASTro, J.M., ArsuagA, J.L., 2012. ESR chronology of alluvial deposits in the Arlanzón valley (Atapuerca, Spain): Contemporaneity with Atapuerca Gran Dolina site. Quaternary Geochronology 10: 418-423. 10.1016/ j.quageo.2012.04.018

Moreno, D., Falguìres, C., PÉrez-GonzÁlez, A., Voinchet, P., Ghaleb, B., Despriée, J., Bahain, J.-J., Sala, R., Carbonell, E., BermúdeZ DE Castro, J.M., Arsuaga, J.L., 2015. New radiometric dates on the lowest stratigraphical section (TD1 to TD6) of Gran Dolina site (Atapuerca, Spain). Quaternary Geochronology 30: 535-540. 10.1016/j.quageo.2015.05.007

Mosquera, M., Saladié, P., Ollé, A., Cáceres, I., HuGuet, R., Villalaín, J.J., Carrancho, A., BourLÈs, D., Braucher, R., Vallverdú, J., 2015. Barranc de la Boella (Catalonia, Spain): an
Acheulean elephant butchering site from the European late Early Pleistocene. Journal of Quaternary Science 30 (7): 651-666. 10.1002/jqs.2800

NonN, H., 1966. Les Régions Côtières de la Galice (Espagne). Étude géomorphologique. Faculté de Lettres, Université de Strasbourg.

NonN, H., 1967. Presentación de algunos depósitos superficiales recientes en Galicia occidental. Notas y comunicaciones del Instituto Geológico y Minero de España 95: 89-105.

NonN, H., Medus, J., 1963. Primeros resultados geomorfológicos y palinológicos referentes a la cuenca de Puentes de García Rodríguez (Galicia). Notas y comunicaciones del Instituto Geológico y Minero de España 71: 87-94.

Ollé, A., Mosquera, M., RodríGuez, X.P., DE LOMBeraHERmida, A., GARCÍA-ANTÓN, M.D., GARcÍAMedrano, P., Peña, L., Menéndez, L., NAVAzo, M., Terradillos, M., Bargalló, A., Márquez, B., Sala, R., Carbonell, E., 2013. The Early and Middle Pleistocene technological record from Sierra de Atapuerca (Burgos, Spain). Quaternary International 295: 138-167. 10.1016/j.quaint.2011.11.009

Parés, J.M., Álvarez, C., Sier, M., Moreno, D., Duval, M., Woodhead, J.D., Ortega, A.I., CAMPaña, I., Rosell, J., Bermúdez de Castro, J.M., CARBONELL, E., 2018. Chronology of the cave interior sediments at Gran Dolina archaeological site, Atapuerca (Spain). Quaternary Science Reviews 186: 1 -16. 10.1016/j.quascirev.2018.02.004

PERLES, C., 1991. Economie des matieres premieres et economie du debitage, deux conceptions opposees, 25 ans d'études technologiques en Préhistoire $X I^{e}$ Rencontres Internationales d'Archéologie et d'Histoire d'Antibes. Éditions APDCA, Juan-les-Pins: 35-45.

Potts, R., Behrensmeyer, A.K., Ditchfield, P., 1999. Paleolandscape variation and Early Pleistocene hominid activities: Members 1 and 7, Olorgesailie Formation, Kenya. Journal of Human Evolution 37 (5): 747-788. 10.1006/jhev.1999.0344

Proença Cunha, P., Martins, A.A., Porto Gouveia, M., 2016. As escadarias de terraços do Ródão à Chamusca (Baixo Tejo) - caracterização e interpretação de dados sedimentares, tectónicos, climáticos e do Paleolítico. Estudos do Quaternario 14: 1-24.

RAPOSO, L., SANTONJA, M., 1995. The earliest occupation of Europe: the Iberian peninsula, en: Roebroeks, W., Kolfschoten, T.V. (Eds.), The earliest occupation of Europe. University of Leiden: 7-25.

Rosina, P., Voinchet, P., Bahain, J.-J., CRistovão, J., FALGUERES, C., 2014. Dating the onset of Lower Tagus River terrace formation using electron spin resonance. Journal of Quaternary Science 29 (2): 153-162. 10.1002/jqs.2689

Rubio-Jara, S., PANera, J., RodrígueZ-DE-TembleQue, J., Santonja, M., PÉrez-GonZÁlez, A., 2016. Large flake Acheulean in the middle of Tagus basin (Spain): Middle stretch of the river Tagus valley and lower stretches of the rivers Jarama and Manzanares valleys. Quaternary International 411: 349 -366. 10.1016/j.quaint.2015.12.023.

Rubio-Jara, S., PANera, J., SANTONJA, M., PÉREZGONZÁlEZ, A., YRAVEDRA, J., DOMÍNGUEZRodrigo, M., Bello, P., Rojas, R., Mabulla, A., 
BAQUEDANO, E., 2017. Site function and lithic technology in the Acheulean technocomplex: a case study from Thiongo Korongo (TK), Bed II, Olduvai Gorge, Tanzania. Boreas. 10.1111/bor.12275

SANTONJA, M., 1992. La adaptación al medio en el Paleolítico Inferior de la Península Ibérica. Elementos para una reflexión, en: Moure Romanillo, J.A. (Ed.), Elefantes, ciervos y ovicrapinos: economía y aprovechamiento del medio en la prehistoria de España y Portugal: $37-76$.

SANTONJA, M., 1996. The Lower Palaeolithic in spain: sites, raw materials an occupation of the land, en: Moloney, N., Raposo, L., Santonja , M. (Eds.), Non-Flint Stone Tools and the Palaeolithic Occupation of the Iberian Peninsula: 1-20.

SAntonja, M., PÉREZ-GonZÁlez, A., 2010. MidPleistocene Acheulean industrial complex in the Iberian Peninsula. Quaternary International 223224: 154-161.

Santonja, M., Pérez-González, A., Panera, J., RubioJARA, S., MÉnDEZ-QuinTAS, E., 2016a. The coexistence of Acheulean and Ancient Middle Palaeolithic techno-complexes in the Middle Pleistocene of the Iberian Peninsula. Quaternary International 411: 367-377. 10.1016/j.quaint.2015.04.056

SANTONJA, M., QUEROL , M.A., 1980. La industria achelense de los niveles fluviales de Áridos, en: Santonja Gómez, M., López Martínez, L., Pérez-González, A. (Eds.), Ocupaciones achelenses en el Valle del Jarama (Arganda, Madrid). Diputación Provincial, Servicios de Extensión Cultural y Divulgación: 231251.

SAntonJA, M., Querol, M.A., 1984. La cultura material durante el Paleolítico Inferior, en: Cano Herrera, M., Chapa Brunet, T., Delibes de Castro, G., Moure Romanillo, A., Querol , M.A., Santonja , M. (Eds.), Prehistoria. Ediciones Nájera: 25-105.

SAntonja, M., Rubio Jara, S., PANera, J., SÁnchezRomero, L., TArriño, A., PÉrez-GonZÁlez, A., 2016b. Ambrona revisited: The Acheulean lithic industry of the Lower Stratigraphic Complex. Quaternary International 480: 95-117. 10.1016/ j.quaint.2017.01.021

SAntonJA, M., Villa, P., 1990. The Lower Paleolithic of Spain and Portugal. Journal of World Prehistory 4 (1): 45-94. 10.1007/bf00974819

Santonja, M., Villa, P., 2006. The Acheulean in Southwestern Europe, en: Goren-Inbar, N., Sharon , G. (Eds.), Axe Age. Acheulian Tool-making from Quarry to Discard. Equinox Publishers: 429-478.

Scott, G.R., GiBert, L., 2009. The oldest hand-axes in Europe. Nature 461 (7260): 82-85.

SHARON, G., 2010. Large flake Acheulian. Quaternary International 223-224: 226-233.

Sharon, G., BARSKY, D., 2016. The emergence of the Acheulian in Europe - A look from the east. Quaternary International 411: 25-33. 10.1016/ j.quaint.2015.11.108

Silva, P.G., Roquero, E., LóPez-Recio, M., Huerta, P., MartíneZ-Graña, A.M., 2017. Chronology of fluvial terrace sequences for large Atlantic rivers in the Iberian Peninsula (Upper Tagus and Duero drainage basins, Central Spain). Quaternary Science Reviews 166: 188-203. 10.1016/ j.quascirev.2016.05.027
Sos BAYNAT, V., 1965. Geomorfología del Valle del Louro, Porriño-Tuy (Pontevedra). Boletín del Instituto Geológico y Minero LXXXVI: 307-355.

TEXIER, J.-P., 1983-1984. Le site paléolithique de Budiño. Portugalia. Nova Serie IV/V: 34-35.

TeXier, J.-P., Cunha Ribeiro, J.-P., Meireles, J., 1995. L'Acheuléen du nord et du centre du Portugal: bilan des connaissances actuelles. Paleo. supplément 1 (1): 185-193.

TEXIER, J.-P., VIDAL ENCINAS, J.M., 1981. Nuevas excavaciones arqueológicas en las Gándaras de Budiño (Pontevedra, Galicia): primeros resultados. I. La estratigrafia, en: Díaz del Olmo, F., Marquez Fernández, D., Rubio Recio, J.M. (Eds.), Vºunion del grupo español de trabajo del Cuaternario, Sevilla, pp. 210-220.

TIXIER, J., 1956. Le hachereau dans l'Acheuléen nordafricain. Notes typologiques, XVe session du Congrès préhistorique de France, Poitiers-Angoulême, pp. 914-923.

Toro-Moyano, I., Barsky, D., Cauche, D., Celiberti, V., Grégoire, S., Lebegue, F., Moncel, M.H., DE LUMLEY, H., 2011. The archaic stone tool industry from Barranco León and Fuente Nueva 3, (Orce, Spain): Evidence of the earliest hominin presence in southern Europe. Quaternary International 243 (1): 80-91. 10.1016/j.quaint.2010.12.011

Vallverdu, J., Saladie, P., Rosas, A., Huguet, R., Caceres, I., Mosquera, M., GarciaTABERnERo, A., Estalrrich, A., LOZANOFernandez, I., Pineda-Alcala, A., CarranCho, A., Villalain, J.J., Bourles, D., BrauCHer, R., Lebatard, A., Vilalta, J., EstebanNADAL, M., BENNASAR, M.L., BASTIR, M., Lopez-Polin, L., Olle, A., Verges, J.M., RosMontoya, S., Martinez-Navarro, B., Garcia, A., Martinell, J., Exposito, I., Burjachs, F., Agusti, J., Carbonell, E., 2014. Age and date for early arrival of the Acheulian in Europe (Barranc de la Boella, la Canonja, Spain). PLOS $O N E$ (7): $9103634.10 .1371 /$ journal.pone. 0103634

VÁZQUeZ VARELA, J.M., 1973. Contribución al estudio del paleolítico gallego. La comarca de río Arenteiro. Cuadernos de Estudios Gallegos XXVIII: 13-22.

VÁZQUez VARELA, J.M., 1984. Paleolítico y mesolítico en Galicia : estado actual de la cuestion : problemas y perspectivas. Portugalia. Nova Serie IV/V: 21-25.

VIDAL ENCINAS, J.M., 1982a. Las Gándaras de Budiño: balance preliminar de dos campañas de excavaciones (1980-1981). El Museo de Pontevedra XXXVI: 91-114.

VIDAL EnCINAS, J.M., 1982b. O Paleolítico Inferior no Baixo Miño marxe galega: unha aproximación. Brigantium 3: 7-32.

VIDAL ENCINAS, J.M., 1983. El yacimiento achelense de las Gandaras de Budiño la colección Pelayo Fernández. Estudio tipológico de bifaces, cantos trabajados y hendedores, Actas del II Seminario de Arqueología del Noroeste: 17-38.

Vieira, M., Poças, E., Pais, J., Pereira, D., 2011. Pliocene flora from S. Pedro da Torre deposits (Minho, NW Portugal). Geodiversitas 33 (1): 71-85. 10.5252/ g2011n1a5 
Eduardo Méndez-Quintas, Manuel Santonja , Alfredo Pérez-González, Lee J. Arnold, João Pedro Cunha Ribeiro, Martina Demuro, Mikel Díaz-Rodríguez, Mathieu Duval, Alberto Gomes, José Meireles, Sérgio Monteiro-Rodrigues \& Andrea Serodio Domínguez

ViVeen, W., Braucher, R., Bourlès, D., SCHOORL, J.M., VELDKAMP, A., VAN BALEN, R.T., WALlinga, J., FERNANDEZ-MOSQUERA, D., VIDAL-ROMANI, J.R., SANJURJO-SANCHEZ, J., 2012. A 0.65Ma chronology and incision rate assessment of the NW Iberian Miño River terraces based on $10 \mathrm{Be}$ and luminescence dating. Global and Planetary Change 94-95: 82-100. 10.1016/j.gloplacha.2012.07.001

VV.AA, 1979. Prehistoria e Arqueoloxía de Galicia. Estado da cuestión. Sección de Arqueoloxía e Prehistoria do Instituto de Estudos Galegos "P. Sarmiento".
Walker, M.J., López-Martínez, M., CARrión-García, J.S., RodRÍGUEZ-ESTRELla, T., SAN-NiCOLÁS DELTORO, M., SCHWENNINGER, J.L., LÓPEZ-JIMÉNEZ, A., Ortega-RodrigáÑez, J., Haber-Uriarte, M., Polo-Camacho, J.L., García-Torres, J., CamPILlo-BoJ, M., AVILÉS-FERnÁNDEZ, A., ZACK, W., 2013. Cueva Negra del Estrecho del Río Quípar (Murcia, Spain): A late Early Pleistocene hominin site with an "Acheulo-Levalloiso-Mousteroid" Palaeolithic assemblage. Quaternary International 294: 135-159. 10.1016/j.quaint.2012.04.038 